\title{
Nitrogen and Phosphorus Fertilization Promotes Aerial Part Development and Affect Nutrient Uptake by Carobinha of the Brazilian Cerrado
}

\author{
Willian Vieira Gonçalves ${ }^{1 *}$, Maria do Carmo Vieira1, Thiago de Oliveira Carnevali², \\ Néstor A. Heredia Zárate1, Heldo Denir Vhaldor Rosa Aran¹, Kátia Cristina Silva Mineli ${ }^{3}$ \\ ${ }^{1}$ Agrarian Science Faculty, Federal University of Grande Dourados-UFGD, University City, Dourados, Brazil \\ ${ }^{2}$ Federal University of Pará-UFPA, São Sebastião, Brazil \\ ${ }^{3}$ Biological and Environmental Sciences Faculty, UFGD, Dourados, Brazil \\ Email: *willianvgoncalves@hotmail.com
}

How to cite this paper: Gonçalves, W.V., do Carmo Vieira, M., de Oliveira Carnevali, T., Zárate, N.A.H., Aran, H.D.V.R. and Mineli, K.C.S. (2017) Nitrogen and Phosphorus Fertilization Promotes Aerial Part Development and Affect Nutrient Uptake by Carobinha of the Brazilian Cerrado. American Journal of Plant Sciences, 8, 3377-3398.

https://doi.org/10.4236/ajps.2017.813227

Received: October 3, 2017

Accepted: December 16, 2017

Published: December 19, 2017

Copyright $\odot 2017$ by authors and Scientific Research Publishing Inc. This work is licensed under the Creative Commons Attribution International License (CC BY 4.0).

http://creativecommons.org/licenses/by/4.0/

(c) (i) Open Access

\begin{abstract}
Producing Brazilian Cerrado plants, especially ones endangered, is essential for your maintenance. In this way, fertilization is furthermore uncertain. Here, we demonstrate the impact of soil addition of nitrogen $(\mathrm{N}, 4.20,18.90$, $31.50,44.10$ and $59.85 \mathrm{mg} \cdot \mathrm{dm}^{-3}$ ) and phosphorus (P, 9.56, 57.38, 95.62, 133.86 and $181.67 \mathrm{mg} \cdot \mathrm{dm}^{-3}$ ) fertilizers levels on the development and on nutrients uptake by Jacaranda decurrens subsp. symmetrifoliolata (carobinha), species of the Brazilian Cerrado, in a long term pot trial. The $\mathrm{N}$ and $\mathrm{P}$ addition together increased plant height and $\mathrm{N}$ concentration in roots. $\mathrm{N}$ and $\mathrm{P}$ also increased the $\mathrm{P}$ concentration and content on the roots in young plants, but in the older plants, isolated effect of both was stronger than their combined action. The $\mathrm{N}$ addition promoted branching, production of dry leaves and dry xylopodium, contents of $\mathrm{K}, \mathrm{Ca}$ and $\mathrm{P}$ on the leaves, and $\mathrm{N}$ content on the roots. However, the $\mathrm{N}$ reduced xylopodium diameter, leaf area, and $\mathrm{Mg}$ contents in the young plants, but increased them in the older plants. The P addition increased stem diameter and dry biomass, $\mathrm{P}$ concentration and $\mathrm{N}$ content on the leaves, Ca content on the roots and also reduced $\mathrm{N}$ concentration on the leaves. However, the $\mathrm{P}$ addition increased $\mathrm{Mg}$ concentration on the roots in the young plants and reduced it in the older plants. In general, $\mathrm{N}$ levels ranging between $25.69-38.85 \mathrm{mg} \cdot \mathrm{dm}^{-3}$ and P levels between $84.39-109.23$ $\mathrm{mg} \cdot \mathrm{dm}^{-3}$ promote more effectively the plant development. Thus, $\mathrm{N}$ and $\mathrm{P}$ fertilization can promote the aerial development of plant and a differential allocation of nutrients between the carobinha tissues.
\end{abstract}




\section{Keywords}

Jacaranda decurrens Sub sp. symmetrifoliolata, Bignoniaceae, Nitrogen Management, Phosphorus Management

\section{Introduction}

Even though the flora of the Brazilian Cerrado is rich in economically important species, their cultivation is still incipient. An aggravating factor is that many of them are endangered, either due to deforestation or because of indiscriminate exploitation, without an appropriate crop management [1] [2]. The use of the roots of these species in traditional medicine increases their risk of extinction. The subject of study in the present work was the carobinha (Jacaranda decurrens subsp. symmetrifoliolata, Bignoniaceae) [3], an underbush species that occurs exclusively in the south of the Mato Grosso do Sul State [4]. The folk medicine indicates their roots as blood depurative, in the healing of uterine and ovarian wounds, and in the treatment of rheumatic problems and dysentery. Recently reports indicate the anti-inflammatory effect of the carobinha roots and the anti-oxidative, anti-tumoral and anti-obesity activities of its leaves [5] [6] [7].

Soil enrichment with nutrients may promote or delay the development of native species in the Brazilian Cerrado [8]. $\mathrm{N}$ and $\mathrm{P}$ play an important role in such responses [9] [10]. In the weathered soils of this ecosystem, $\mathrm{P}$ is one of the most limiting factors [11]. In the plant, $\mathrm{P}$ is essential in the supply of energy and in the composition of RNA, DNA, and photosynthesis and respiration derived sugars. Besides, it is a crucial component of the cellular membrane [12]. [13] also showed that fertilization with inorganic $\mathrm{P}$ may promote growth and leaf and root production in the carobinha. In turn, $\mathrm{N}$ composes the structure of amino acids and nucleic acids, being quantitatively the nutrient that is most required by plants [12]. In general, $\mathrm{N}$ increases potassium $(\mathrm{K})$ demands and $\mathrm{P}$ uptake in the plant. Furthermore, it increases the amounts of sulfur (S) needed for amino acid synthesis [14]. However, an excessive application of $\mathrm{N}$ may lead to $\mathrm{K}$ deficiency and reduced calcium (Ca) levels [15], and ammonium $\left(\mathrm{NH}_{4}^{+}\right)$addition to the soil may increase its acidity [16]. Therefore, the balanced addition of $\mathrm{N}$ and $\mathrm{P}$ is essential for the correct balancing of plant development and nutrient uptake [17] [18].

Based on the exposed situation, we investigated the effect of soil fertilization with $\mathrm{N}$ and $\mathrm{P}$ on the development and nutrients uptake by the carobinha.

\section{Material and Methods}

\subsection{Protected Growth}

The experiment was carried out between April 2012 and June 2014, under a $2 \mathrm{~m}$ $\times 2 \mathrm{~m}$ (height, width) tunnel with $50 \%$ shading and a plastic roof, locate at $22^{\circ} 11^{\prime} 44.45^{\prime \prime}$ latitude, $54^{\circ} 56^{\prime} 07.31^{\prime \prime} \mathrm{W}$ longitude and $460 \mathrm{~m}$ altitude. The plants 
were cultivated in vases with $5.70 \mathrm{~kg}$ substrate (very clayey dystroferric Red Latosol; $1.20 \mathrm{~kg} \cdot \mathrm{dm}^{-3} ; \mathrm{pH} \mathrm{CaCl}_{2}=4.33 ; P=1.71 \mathrm{mg} \cdot \mathrm{dm}^{-3} ; \mathrm{K}=0.041 \mathrm{cmol}_{c} \cdot \mathrm{dm}^{-3} ; \mathrm{Al}$ $=1.2 \mathrm{cmol}_{c} \cdot \mathrm{dm}^{-3} ; \mathrm{Ca}=0.67 \mathrm{cmol}_{c} \cdot \mathrm{dm}^{-3} ; \mathrm{Mg}=0.18 \mathrm{cmol}_{c} \cdot \mathrm{dm}^{-3} ; \mathrm{H}+\mathrm{Al}=2.99$ $\left.\mathrm{cmol}_{c} \cdot \mathrm{dm}^{-3} ; \mathrm{T}=3.89 \mathrm{cmol}_{c} \cdot \mathrm{dm}^{-3} ; \mathrm{V}=22.9 \%\right)$. After addition of $24.54 \mathrm{~g}$ limestone

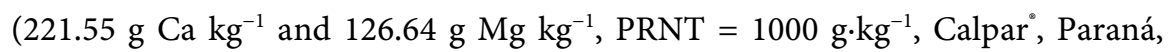
Brazil) and $200 \mathrm{~mL}$ water $\mathrm{kg}^{-1}$ to the substrate, the vases were incubated for 14 days.

Five nitrogen $(\mathrm{N})(\mathrm{N} 1: 4.20, \mathrm{~N} 2: 18.90, \mathrm{~N} 3: 31.50, \mathrm{~N} 4: 44.10$ and N5: 59.85 $\mathrm{mg} \cdot \mathrm{dm}^{-3}$ ) and five phosphorus (P) (P1: 9.56, P2: 57.38, P3: 95.62, P4: 133.86 and P5: $181.67 \mathrm{mg} \cdot \mathrm{dm}^{-3}$ ) fertilizers levels and cycles were tested. Their combinations were defined based on the experimental matrix Plan Plueba III, which suggested nine treatments (1: N2P2, 2: N4P2, 3: N2P4, 4: N4P4, 5: N3P3, 6: N1P2, 7: N5P4, 8: N2P1 and 9: N4P5) arranged in randomized blocks and four repetitions. The experimental unit consisted of four vases. Phosphorus was provided by $\mathrm{Ca}_{2}\left(\mathrm{H}_{2} \mathrm{PO}_{4}\right)\left(187.7 \mathrm{~g} \mathrm{P} \mathrm{kg}^{-1}+150 \mathrm{~g} \mathrm{Ca} \mathrm{kg}^{-1}\right)$, nitrogen by $\left(\mathrm{NH}_{4}\right)_{2} \mathrm{SO}_{4}\left(210 \mathrm{~g} \mathrm{~N} \mathrm{~kg}^{-1}\right.$ $\left.+220 \mathrm{~g} \mathrm{~S} \mathrm{~kg}^{-1}\right)$, and potassium $\left(120 \mathrm{mg} \cdot \mathrm{dm}^{-3}\right)$ by $\mathrm{KCl}\left(498.1 \mathrm{~g} \mathrm{~K} \mathrm{~kg}^{-1}\right)$.

After incubation, one 70-day-old carobinha plantlet was transferred to each vase. Total $\mathrm{P}$ was applied on the same day; $40 \% \mathrm{~N}$ and $\mathrm{K}$ were added 30 days later; and the remaining was applied 60 (30\%) and 90 (30\%) days after transplanting (DAT). At 151 DAT, $0.15 \mathrm{~mL}$ of a $1.2 \mathrm{~kg} \mathrm{~L}^{-1}$ fertilization solution $(60 \mathrm{~g} \mathrm{Ca}$ $\mathrm{L}^{-1}, 12 \mathrm{~g} \mathrm{Mg} \mathrm{L}^{-1}, 24 \mathrm{~g} \mathrm{~S} \mathrm{~L}^{-1}, 2.4 \mathrm{~g} \mathrm{~B} \mathrm{~L}^{-1}, 3 \mathrm{~g} \mathrm{Cu} \mathrm{L}^{-1}, 12 \mathrm{~g} \mathrm{Mn} \mathrm{L}^{-1}, 0.432 \mathrm{~g} \mathrm{Mo} \mathrm{L}^{-1}$ and $12 \mathrm{~g} \mathrm{Zn} \mathrm{L}^{-1}$; Supra Mix ${ }^{\oplus}$, Supra Fertilizantes) were added to each vase. The substrate was kept at $70 \%$ of the field capacity.

Plant height and stem diameter were measured during the experimental cycle (between 44 and $672 \mathrm{DAT}$ ). At $259 \mathrm{DAT}$ and $770 \mathrm{DAT}$, two entire plants were harvested in order to determinate xylopodium diameter; number of leaves and branches; leaf area; and the production of leaves, stems, xylopodium, and fresh and dry roots. The material was dried in a drying oven with forced air ventilation, at $60^{\circ} \mathrm{C} \pm 5^{\circ} \mathrm{C}$, until it reached a constant weight.

\subsection{Chemical Analyses of Soils and Plants}

The substrate collected before correction and at $259 \mathrm{DAT}$ was dried, under natural conditions, grinded and sieved $(<2 \mathrm{~mm})$. Then, were determined the $\mathrm{pH}$ $\left(\mathrm{CaCl}_{2}\right), \mathrm{H}+\mathrm{Al}$ [SMP method, $\ln (\mathrm{H}+\mathrm{Al})=8.0857763-1.0621553 \times \mathrm{pH}$ SMP], $\mathrm{K}$ (flame photometry, Melich 1), $\mathrm{Ca}$ and $\mathrm{Mg}$ (atomic absorption spectrophotometry-AAS, Varian SpectrAA-240 FS, Varian), Al (volumetry) and P (spectrophotometry, Melich 1). Sum of bases $(\mathrm{Ca}+\mathrm{Mg}+\mathrm{K})$, cation exchange capacity (CEC) (sum of bases $+\mathrm{H}+\mathrm{Al}$ ) and base saturation (sum of basis/CEC) were also calculated.

The leaves and roots from each sample were grinded using a knife grinder type Willye $(0.841 \mathrm{~mm})$. K (flame photometry), $\mathrm{Ca}$ and $\mathrm{Mg}$ (atomic absorption spectrophotometry), total $\mathrm{N}$ (Kjeldahl acid digestion) and $\mathrm{P}$ (spectrophotometry) were determined. The nutrient contents in the tissues were calculated by multiplying the nutrient concentration by the dry mass. 


\subsection{Statistical Analyses}

The open source software R [19] and Origin (OriginLab, Northampton, MA) were used to carry out the statistical analyses. The data vignette of $\mathrm{R}$ was stored in the EACS package [20]. The residues were initially tested for normality; if they did not show a normal distribution, the data were transformed using the boxcox test (MASS package) [21] in order to maximize their normality. Table 1 is all transformations. We then tested the constant effect of the treatments on the dependent variables, with the random effect of the block per experimental unit, compared to the model with the random effect of the block (lme, nlme package) [22]. The same model was applied to test the combined effect of $\mathrm{N}$ and $\mathrm{P}$ on plant age, considering age as variable.

Without the effect of the experimental unit, the linear models were tested (lm, lattice package) [23]. We tested the effect of nitrogen and phosphorus on nutrient concentration in the soil and on the leaves at $259 \mathrm{DAT}$, since at $770 \mathrm{DAT}$ the amount of leaves was insufficient. The repetitions were therefore combined and only the effect of age at harvest was tested. Wireframe plots (lattice package) were used to build response surfaces and boxplots (ggplot2) were used to represent the graphs showing the effect of age at harvest [24]. The logistic nonlinear model adjusted itself to the effect of evaluation time on plant height, and, the modified Hill equation [25], to the effect of plant age on stem diameter. The repetitions were taken into account in the regressions, therefore, the coefficients of determination $\left(\mathrm{R}^{2}\right)$ are multiple.

\section{Results and Discussion}

\subsection{Effect of $\mathrm{N}$ and $\mathrm{P}$ Fertilization on Soil Chemistry}

Both $\mathrm{N}(p=0.10)$ and $\mathrm{P}(p=0.52)$ had no effect on soil $\mathrm{pH}\left(\mathrm{CaCl}_{2}\right)$. The $\mathrm{N}$ addition elevated of $\mathrm{K}$ contents $(p=0.01)$ and slight increased the levels of $\mathrm{H}+\mathrm{Al}(p$ $=0.07)$. Intermediate $\mathrm{N}$ doses $\left(24.85 \mathrm{mg} \cdot \mathrm{dm}^{-3}\right)$ increased $\mathrm{K}$ levels to highest $\left(0.34 \mathrm{cmolc} \cdot \mathrm{dm}^{-3}\right)$ (maximum P), Figure 1(a). The regression models were not adjusted to the effect of $\mathrm{N}$ on $\mathrm{H}+\mathrm{Al}\left(\mathrm{R}^{2}, p=0.59\right)$. The $\mathrm{N}$ addition elevated of $\mathrm{H}$ $+\mathrm{Al}$ levels up to $31.5 \mathrm{mg} \cdot \mathrm{dm}^{-3}$ but, as doses increased, the levels drastically decreased. The $\mathrm{P}$ addition affected $\mathrm{Mg}$ levels $(p=0.01)$ and the sum of bases $(p=$ 0.02 and $p=0.02$ ), and more slightly Ca contents $(p=0.04$ and $p=0.07)$, CEC $(p=0.02$ and $p=0.10)$ and base saturation $(p=0.05$ and $p=0.09)$. However, the regression models were not adjusted to the effect of $\mathrm{P}$ on $\mathrm{Ca}\left(\mathrm{R}^{2}, p=0.23\right)$, the sum of bases $\left(\mathrm{R}^{2}, p=0.11\right.$ and $\left.p=0.15\right)$, the $\mathrm{CEC}\left(\mathrm{R}^{2}, p=0.12\right)$ and the base saturation $\left(\mathrm{R}^{2}, p=0.16\right)$. The proportional increase of $\mathrm{P}$ doses elevated the $\mathrm{Mg}$ contents up to a maximum of $\left(5.39 \mathrm{cmol}_{c} \cdot \mathrm{dm}^{-3}\right)$ (minimum N), Figure $1(\mathrm{~b})$. The addition of $\mathrm{N}$ and $\mathrm{P}$ affected the $\mathrm{P}$ levels $(\mathrm{N}, p<0.01$ and $p=0.03$, and $\mathrm{P}, p<$ 0.01 and $p=0.01)$ and the $\mathrm{P}$ recovery efficiency $(\mathrm{N}, p=0.04$ and $\mathrm{P}, p=0.01)$. Soil supplementation with $\mathrm{P}$ (from $11.42 \mathrm{mg} \cdot \mathrm{dm}^{-3}$ until a maximum dose) and of $\mathrm{N}$ (until $19.32 \mathrm{mg} \cdot \mathrm{dm}^{-3}$ ) resulted in the highest $\mathrm{P}$ contents $\left(38.56 \mathrm{mg} \cdot \mathrm{dm}^{-3}\right)$, Figure $1(\mathrm{c})$. However, $\mathrm{N}$ and $\mathrm{P}$ had opposite effects on the $\mathrm{P}$ recovery efficiency: 
Table 1. Regression equations of fitted models for plant age at evaluation or harvest $(\mathrm{H})$, nitrogen $(\mathrm{N})$ and phosphorus (P) effects.

\begin{tabular}{|c|c|c|c|}
\hline $\begin{array}{c}\text { Dependent } \\
\text { variable }(\mathrm{dv})\end{array}$ & $\begin{array}{l}\text { Transformation } \\
\text { for Normality }\end{array}$ & Fitted models & $\begin{array}{l}\text { Multiple } \\
\mathrm{R}^{2}\end{array}$ \\
\hline \multicolumn{4}{|c|}{ Soil chemistry } \\
\hline $\mathrm{K}$ & $\mathrm{dv}^{-0.41}$ & $\begin{array}{l}\hat{\mathrm{y}}=-0.041849^{(p=0.730)} \mathrm{b} 1-0.0816^{(p=0.044)} \mathrm{b} 2-0.044357^{(p=0.276)} \mathrm{b} 3 \\
-0.052133^{(p=0.189)} \mathrm{b} 4+0.116161^{(p=0.018)} \sqrt{\mathrm{N}}+0.007102^{(p=0.205)} \sqrt{\mathrm{P}}-0.01165^{(p=0.019)} \mathrm{N}\end{array}$ & $0.359^{(p=0.039)}$ \\
\hline $\mathrm{Mg}$ & - & $\begin{array}{l}\hat{\mathrm{y}}=4.879078^{(p<0.000)} \mathrm{b} 1-0.155711^{(p=0.228)} \mathrm{b} 2-0.027805^{(p=0.833)} \mathrm{b} 3 \\
-0.022856^{(p=0.858)} \mathrm{b} 4-0.008871^{(p=0.010)} \mathrm{N}+0.003007^{(p=0.008)} \mathrm{P}\end{array}$ & $0.291^{(p=0.063)}$ \\
\hline $\mathrm{P}$ & $\mathrm{dv}^{0.46}$ & $\begin{array}{l}\hat{\mathrm{y}}=0.83309^{(p=0.911) \ddagger} \mathrm{b} 1^{\mathrm{a}}+2.38973^{(p=0.182)} \mathrm{b} 2+1.8747^{(p=0.307)} \mathrm{b} 3+1.1502^{(p=0.516)} \mathrm{b} 4 \\
+2.27874^{(p=0.295)} \sqrt{\mathrm{N}}-2.44087^{(p=0.033)} \sqrt{\mathrm{P}}-0.25923^{(p=0.237)} \mathrm{N}+0.36121^{(p<0.000)} \mathrm{P}\end{array}$ & $0.922^{(p<0.000)}$ \\
\hline $\begin{array}{c}\mathrm{P} \\
\text { expected/P soil }\end{array}$ & - & $\begin{array}{l}\hat{\mathrm{y}}=0.2687536^{(p=0.009)} \mathrm{b} 1+0.0353915^{(p=0.125)} \mathrm{b} 2+0.0289716^{(p=0.220)} \mathrm{b} 3+0.0173949^{(p=0.444)} \mathrm{b} 4 \\
+0.0394384^{(p=0.161)} \sqrt{\mathrm{N}}-0.0514625^{(p=0.001)} \sqrt{\mathrm{P}}-0.0038727^{(p=0.170)} \mathrm{N}+0.0029828^{(p=0.001)} \mathrm{P}\end{array}$ & $0.454^{(p=0.013)}$ \\
\hline \multicolumn{4}{|c|}{ Growth and development } \\
\hline $\begin{array}{l}\text { Plant } \\
\text { height }\end{array}$ & $\mathrm{dv}^{-0.19}$ & $\begin{array}{l}\mathrm{N} \text { and P effects: } \hat{y}=5.425592^{(p=0.005)}+0.413743^{(p=0.361)} \sqrt{\mathrm{N}}+0.653603^{(p=0.008)} \sqrt{\mathrm{P}} \\
-0.144198^{(p=0.037)} \mathrm{N}-0.055969^{(p=0.008)} \mathrm{P}+0.101196^{(p=0.177)} \sqrt{\mathrm{N}} * \sqrt{\mathrm{P}} \\
\text { Plant age effect: } \hat{y}=2.91918+\left(2.91918-15.23677 / 1+(x / 204.283)^{9.23491}\right)\end{array}$ & $\begin{array}{c}- \\
0.694 \\
\left(X^{2}=12.534, \mathrm{k}=524\right)\end{array}$ \\
\hline $\begin{array}{c}\text { Stem } \\
\text { diameter }\end{array}$ & $\mathrm{dv}^{-0.05}$ & $\begin{array}{l}\mathrm{N} \text { and P effects: } \hat{\mathrm{y}}=2.4122229^{(p=0.026)}+0.1398655^{(p=0.629)} \sqrt{\mathrm{N}}+0.5901713^{(p=0.000)} \sqrt{\mathrm{P}} \\
-0.0189229^{(p=0.515)} \mathrm{N}-0.0254747^{(p=0.004)} \mathrm{P} \\
\text { Plant age effect: } \hat{y}=1.29818+(8.80959-1.29818) \times \frac{x^{7.83389}}{224.89332^{7.83389}+x^{7.83389}}\end{array}$ & $\begin{array}{c}- \\
0.681 \\
\left(X^{2}=4.570, \mathrm{k}=536\right)\end{array}$ \\
\hline Branching & $\mathrm{dv}^{0.10}$ & $\begin{array}{l}\hat{\mathrm{y}}=1.624617^{(p=0.101)} \mathrm{b} 1+0.657073^{(p=0.465)} \mathrm{b} 2-0.845044^{(p=0.362)} \mathrm{b} 3+0.157073^{(p=0.861)} \mathrm{b} 4 \\
+0.052074^{(p=0.024)} \mathrm{N}-0.009139^{(p=0.218)} \mathrm{P}+3.281516^{(p<0.000)} \mathrm{H}^{\mathrm{b}}\end{array}$ & $\begin{array}{c}0.364 \\
(p=0.000)\end{array}$ \\
\hline $\begin{array}{l}\text { Xylopodium } \\
\text { diameter }\end{array}$ & $\mathrm{dv}^{0.10}$ & $\begin{array}{l}\hat{\mathrm{y}}=12.44178^{(p=0.001)} \mathrm{b} 1-1.027^{(p=0.230)} \mathrm{b} 2-1.88898^{(p=0.035)} \mathrm{b} 3-2.47894^{(p=0.005)} \mathrm{b} 4 \\
-1.78980^{(p=0.207)} \sqrt{\mathrm{N}}+0.04876^{(p=0.771)} \sqrt{\mathrm{P}}+0.20118^{(p=0.162)} \mathrm{N}-5.07082^{(p=0.325)} \mathrm{H} \\
+5.49379^{(p=0.008)} \sqrt{\mathrm{N}}{ }^{*} \mathrm{H}+0.09654^{(p=0.684)} \sqrt{\mathrm{P}}{ }^{*} \mathrm{H}-0.56656^{(p=0.007)} \mathrm{N}^{\star} \mathrm{H}\end{array}$ & $\begin{array}{c}0.744 \\
(p<0.000)\end{array}$ \\
\hline Leaf area & $\mathrm{dv}^{0.35}$ & $\begin{array}{l}\hat{\mathrm{y}}=465.37492^{(p<0.000)} \mathrm{b} 1-49.56625^{(p=0.247)} \mathrm{b} 2-69.63742^{(p=0.116)} \mathrm{b} 3-68.42314^{(p=0.112)} \mathrm{b} 4 \\
-10.62847^{(p=0.044)} \mathrm{N}+0.31991^{(p=0.518)} \mathrm{P}+0.20204^{(p=0.011)} \mathrm{N}^{2}-416.06656^{(p=0.000)} \mathrm{H} \\
+14.188^{(p=0.061)} \mathrm{N}{ }^{\star} \mathrm{H}-0.40932^{(p=0.559)} \mathrm{P}{ }^{\star} \mathrm{H}-0.25522^{(p=0.025)} \mathrm{N}^{2 \star} \mathrm{H}\end{array}$ & $\begin{array}{c}0.705 \\
(p<0.000)\end{array}$ \\
\hline Dry leaves & $\mathrm{dv}^{0.50}$ & $\begin{array}{l}\hat{\mathrm{y}}=8.628721^{(p<0.000)} \mathrm{b} 1-0.091967^{(p=0.893)} \mathrm{b} 2-0.740256^{(p=0.297)} \mathrm{b} 3-0.811167^{(p=0.239)} \mathrm{b} 4 \\
-0.152607^{(p=0.071)} \mathrm{N}-0.005056^{(p=0.526)} \mathrm{P}+0.003567^{(p=0.006)} \mathrm{N}^{2}-8.059523^{(p<0.000)} \mathrm{H} \\
+0.183065^{(p=0.131)} \mathrm{N}^{\star} \mathrm{H}+0.003525^{(p=0.754)} \mathrm{P}{ }^{\star} \mathrm{H}-0.003959^{(p=0.031)} \mathrm{N}^{2} \mathrm{H}\end{array}$ & $\begin{array}{c}0.798 \\
(p<0.000)\end{array}$ \\
\hline Dry stems & $\mathrm{dv}^{0.40}$ & $\begin{array}{l}\hat{\mathrm{y}}=0.67805^{(p=0.654)} \mathrm{b} 1-0.3142^{(p=0.376)} \mathrm{b} 2-0.70753^{(p=0.056)} \mathrm{b} 3-0.67275^{(p=0.061)} \mathrm{b} 4 \\
-0.2451^{(p=0.573)} \sqrt{\mathrm{N}}+0.48083^{(p=0.033)} \sqrt{\mathrm{P}}+0.03615^{(p=0.407)} \mathrm{N}-0.02617^{(p=0.048)} \mathrm{P}\end{array}$ & $\begin{array}{c}0.201 \\
(p=0.048)\end{array}$ \\
\hline $\begin{array}{l}\text { Dry } \\
\text { xylopodium }\end{array}$ & $\mathrm{dv}^{0.20}$ & $\begin{array}{l}\hat{\mathrm{y}}=0.05681^{(p=0.976)} \mathrm{b} 1+0.948^{(p=0.584)} \mathrm{b} 2-0.68121^{(p=0.702)} \mathrm{b} 3-2.02281^{(p=0.244)} \mathrm{b} 4 \\
+0.05426^{(p=0.216)} \mathrm{N}-0.01043^{(p=0.464)} \mathrm{P}+6.95798^{(\mathrm{p}<0.000)} \mathrm{H}\end{array}$ & $\begin{array}{c}0.373 \\
(p=0.000)\end{array}$ \\
\hline Dry roots & $\mathrm{dv}^{0.20}$ & $\begin{array}{l}\hat{\mathrm{y}}=3.7396659^{(p=0.009)} \mathrm{b} 1-1.4331236^{(p=0.260)} \mathrm{b} 2-1.2369638^{(p=0.345)} \mathrm{b} 3 \\
-1.843158^{(p=0.149)} \mathrm{b} 4+0.0201585^{(p=0.529)} \mathrm{N}+0.0001814^{(p=0.986)} \mathrm{P}+5.1851406^{(p<0.000)} \mathrm{H}\end{array}$ & $\begin{array}{c}0.366 \\
(p=0.000)\end{array}$ \\
\hline \multicolumn{4}{|c|}{ Nutrients concentration and content } \\
\hline $\begin{array}{c}\text { Leaf } \mathrm{P} \\
\text { concentration }\end{array}$ & $\mathrm{dv}^{-0.05}$ & $\begin{array}{l}\hat{\mathrm{y}}=1.602808^{(p<0.000)} \mathrm{b} 1+0.204678^{(p=0.355)} \mathrm{b} 2+0.039961^{(p=0.856)} \mathrm{b} 3 \\
+0.011696^{(p=0.958)} \mathrm{b} 4-0.009975^{(p=0.092)} \mathrm{N}+0.004881^{(p=0.013)} \mathrm{P}\end{array}$ & $\begin{array}{c}0.167 \\
(p=0.194)\end{array}$ \\
\hline $\begin{array}{c}\text { Leaf } \mathrm{N} \\
\text { concentration }\end{array}$ & - & $\begin{array}{l}\hat{\mathrm{y}}=21.25279^{(p=0.001)} \mathrm{b} 1+5.75556^{(p<0.000)} \mathrm{b} 2+3.5^{(p=0.005)} \mathrm{b} 3+3.57778^{(p=0.004)} \mathrm{b} 4 \\
-1.52302^{(p=0.309)} \sqrt{\mathrm{N}}-1.40047^{(p=0.071)} \sqrt{\mathrm{P}}+0.14062^{(p=0.348)} \mathrm{N}+0.08411^{(p=0.066)} \mathrm{P}\end{array}$ & $\begin{array}{l}0.4611 \\
(p=0.001)\end{array}$ \\
\hline
\end{tabular}




\section{Continued}

\begin{tabular}{|c|c|c|c|}
\hline $\begin{array}{l}\text { Root Mg } \\
\text { concentration }\end{array}$ & - & $\begin{array}{l}\hat{\mathrm{y}}=2.056803^{(p=0.000)}+0.0268999^{(p=0.051)} \mathrm{N}+0.0020677^{(p=0.113)} \mathrm{P} \\
-0.0004479^{(p=0.031)} \mathrm{N}^{2}-0.4621669^{(p=0.058)} \mathrm{H}-0.0344858^{(p<0.042)} \mathrm{N}{ }^{\star} \mathrm{H} \\
-0.0001672^{(p<0.042)} \mathrm{P}{ }^{\star} \mathrm{H}+0.0005432^{(p<0.033)} \mathrm{N}^{2} \mathrm{H}\end{array}$ & - \\
\hline $\begin{array}{c}\text { Root } \mathrm{N} \\
\text { concentration }\end{array}$ & $\mathrm{dv}^{0.70}$ & $\begin{array}{l}\hat{\mathrm{y}}=11.3349596^{(p<0.000)} \mathrm{b} 1+0.8571477^{(p=0.353)} \mathrm{b} 2+1.6749204^{(p=0.081)} \mathrm{b} 3 \\
+1.5571477^{(p=0.094)} \mathrm{b} 4+0.0750559^{(p=0.362)} \mathrm{N}-0.0508524^{(p=0.051)} \mathrm{P} \\
-0.0008661^{(p=0.482)} \mathrm{N}^{2}+0.000345^{(p=0.011)} \mathrm{P}^{2}-7.6807986^{(p<0.000)} \mathrm{H}\end{array}$ & $\begin{array}{c}0.726 \\
(p<0.000)\end{array}$ \\
\hline $\begin{array}{c}\text { Root } \mathrm{P} \\
\text { concentration }\end{array}$ & $\mathrm{dv}^{0.10}$ & $\begin{array}{l}\hat{\mathrm{Y}}=2.391^{(p=0.000)} \mathrm{b} 1-0.02556^{(p=0.899)} \mathrm{b} 2+0.08954^{(p=0.667)} \mathrm{b} 3+0.2154^{(p=0.285)} \mathrm{b} 4 \\
-0.007839^{(p=0.754)} \mathrm{N}+0.00002428^{(p=0.998)} \mathrm{P}-0.0004613^{(p=0.411)} \mathrm{N}^{2}-0.00001497^{(p=0.800)} \mathrm{P}^{2} \\
+0.0002969^{(p=0.301)} \mathrm{N} \mathrm{N}^{\star} \mathrm{P}-1.383^{(p=0.050)} \mathrm{H}+0.004393^{(p=0.903)} \mathrm{N}^{\star} \mathrm{H}+0.004635^{(p=0.681)} \mathrm{P}{ }^{\star} \mathrm{H} \\
+0.001117^{(p=0.175)} \mathrm{N}^{2}{ }^{(p-0.292)} \mathrm{H}+0.00009119^{2 \star} \mathrm{P}^{\left(p-0.0006997^{(p=0.093)} \mathrm{N}\right.} \mathrm{P}^{\star} \mathrm{P} \mathrm{H}\end{array}$ & $\begin{array}{c}0.485 \\
(p=0.000)\end{array}$ \\
\hline Leaf $\mathrm{K}$ content & $\mathrm{dv}^{0.31}$ & $\begin{array}{l}\hat{\mathrm{y}}=3.906313^{(p<0.000)} \mathrm{b} 1+0.062593^{(p=0.907)} \mathrm{b} 2-0.248765^{(p=0.642)} \mathrm{b} 3-0.530851^{(p=0.312)} \mathrm{b} 4 \\
+0.029042^{(p=0.031)} \mathrm{N}-0.002014^{(p=0.631)} \mathrm{P}-4.018072^{(\mathrm{p}<0.000)} \mathrm{H}\end{array}$ & $\begin{array}{c}0.712 \\
(p<0.000)\end{array}$ \\
\hline Leaf Ca content & $\mathrm{dv}^{0.29}$ & $\begin{array}{l}62.079^{(\mathrm{p}<0.000)} \mathrm{b} 1-0.98366^{(p=0.905)} \mathrm{b} 2-9.46959^{(p=0.253)} \mathrm{b} 3-10.92011^{(p=0.178)} \mathrm{b} 4 \\
+0.44128^{(p=0.033)} \mathrm{N}-0.02716^{(p=0.6741)} \mathrm{P}-62.04177^{(p<0.000)} \mathrm{H}\end{array}$ & $\begin{array}{c}0.716 \\
(p<0.000)\end{array}$ \\
\hline Leaf $\mathrm{Mg}$ content & $\mathrm{dv}^{0.25}$ & $\begin{array}{l}\hat{\mathrm{y}}=23.13^{(p<0.000)} \mathrm{b} 1-1.396^{(p=0.544)} \mathrm{b} 2-3.124^{(p=0.172)} \mathrm{b} 3-3.109^{(p=0.163)} \mathrm{b} 4-0.4771^{(p=0.052)} \mathrm{N} \\
+0.0003478^{(p=0.988)} \mathrm{P}+0.0103^{(p=0.006)} \mathrm{N}^{2}-0.2101^{(p=0.001)} \mathrm{H}+0.5867^{(p=0.151)} \mathrm{N}{ }^{\star} \mathrm{H} \\
-0.0102^{(p=0.774)} \mathrm{P}{ }^{\star} \mathrm{H}-0.01146^{(p=0.056)} \mathrm{N}^{2} \mathrm{H}\end{array}$ & $\begin{array}{c}0.770 \\
(p<0.000)\end{array}$ \\
\hline Leaf $\mathrm{N}$ content & $\mathrm{dv}^{0.27}$ & $\begin{array}{l}\hat{\mathrm{y}}=133.1^{(p<0.000)} \mathrm{b} 1+9.096^{(p=0.490)} \mathrm{b} 2-16.33^{(p=0.212)} \mathrm{b} 3-15.07^{(p=0.238)} \mathrm{b} 4-3.209^{(p=0.025)} \mathrm{N} \\
+0.2466^{(p=0.573)} \mathrm{P}+0.06748^{(p=0.002)} \mathrm{N}^{2}-0.00137^{(p=0.530)} \mathrm{P}^{2}-117.2^{(p=0.009)} \mathrm{H} \\
+3.385^{(p=0.154)} \mathrm{N}{ }^{\star} \mathrm{H}-0.385^{(p=0.577)} \mathrm{P}{ }^{\star} \mathrm{H}-0.06893^{(p=0.049)} \mathrm{N}^{2} \mathrm{H}+0.001938^{(p=0.572)} \mathrm{P}^{2 \star} \mathrm{H}\end{array}$ & $\begin{array}{c}0.806 \\
(p<0.000)\end{array}$ \\
\hline Leaf $\mathrm{P}$ content & $\mathrm{dv}^{0.25}$ & $\begin{array}{l}\hat{\mathrm{y}}=10.948408^{(p=0.007)}-0.319135^{(p=0.120)} \mathrm{N}+0.099322^{(p=0.123)} \mathrm{P}+0.006029^{(p=0.053)} \mathrm{N}^{2} \\
-0.000384^{(p=0.227)} \mathrm{P}^{2}-9.793508^{(p=0.122)} \mathrm{H}+0.3227^{(p=0.336)} \mathrm{N}{ }^{*} \mathrm{H} \\
-0.111079^{(p=0.260)} \mathrm{P}^{\star} \mathrm{H}-0.005992^{(p=0.226)} \mathrm{N}^{2}{ }^{\star} \mathrm{H}+0.000449^{(p=0.357)} \mathrm{P}^{2}{ }^{*} \mathrm{H}\end{array}$ & - \\
\hline Root Ca content & $\mathrm{dv}^{0.08}$ & $\begin{array}{l}\hat{\mathrm{y}}=10.33276^{(p=0.704)} \mathrm{b} 1-5.67455^{(p=0.224)} \mathrm{b} 2-8.03203^{(p=0.096)} \mathrm{b} 3-9.28819^{(p=0.049)} \mathrm{b} 4 \\
-1.4206^{(p=0.857)} \sqrt{\mathrm{N}}+1.44502^{(p=0.719)} \sqrt{\mathrm{P}}+0.20271^{(p=0.798)} \mathrm{N}-0.09138^{(p=0.701)} \mathrm{P} \\
+44.28281^{(p=0.262)} \mathrm{H}-2.66291^{(p=0.815)} \sqrt{\mathrm{N}}{ }^{\star} \mathrm{H}-8.87399^{(p=0.129)} \sqrt{\mathrm{P}}{ }^{*} \mathrm{H} \\
+0.14825^{(p=0.897)} \mathrm{N}{ }^{*} \mathrm{H}+0.62855^{(p=0.070)} \mathrm{P}{ }^{\star} \mathrm{H}\end{array}$ & $\begin{array}{l}0.3333 \\
(p=0.017)\end{array}$ \\
\hline Root $\mathrm{P}$ content & $\mathrm{dv}^{0.21}$ & $\begin{array}{l}\hat{\mathrm{y}}=9.5097314^{(p=0.141)} \mathrm{b} 1-1.8589245^{(p=0.474)} \mathrm{b} 2-3.1484625^{(p=0.240)} \mathrm{b} 3-2.8415475^{(p=0.275)} \mathrm{b} 4 \\
-0.1465441^{(p=0.645)} \mathrm{N}+0.0347105^{(p=0.728)} \mathrm{P}+0.0002882^{(p=0.968)} \mathrm{N}^{2}-0.0003529^{(p=0.638)} \mathrm{P}^{2} \\
+0.0015151^{(p=0.676)} \mathrm{N}{ }^{*} \mathrm{P}-0.0498683^{(p=0.996)} \mathrm{H}-0.0035868^{(p=0.994)} \mathrm{N}{ }^{*} \mathrm{H} \\
+0.0241799^{(p=0.868)} \mathrm{P}^{\star} \mathrm{H}+0.0137572^{(p=0.200)} \mathrm{N}^{2 \star} \mathrm{H}+0.0016135^{(p=0.154)} \mathrm{P}^{2} \mathrm{H} \\
-0.0090576^{(p=0.091)} \mathrm{N}^{\star} \mathrm{P}{ }^{\star} \mathrm{H}\end{array}$ & $\begin{array}{c}0.358 \\
(p=0.023)\end{array}$ \\
\hline
\end{tabular}

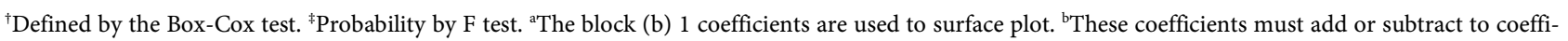
cients of intercept or b1, and respectively effects $\left(\mathrm{N}, \mathrm{P}, \mathrm{P}^{2}, \mathrm{~N}^{2}, \sqrt{\mathrm{N}}, \sqrt{\mathrm{P}}\right.$ and their interactions) on harvest of 770 DAT.

intermediate $\mathrm{N}$ doses increased, while $\mathrm{P}$ doses reduced it. The lower $\mathrm{N}$ dose and $74.42 \mathrm{mg} \cdot \mathrm{dm}^{-3} \mathrm{P}$ resulted in the lowest recovery efficiency $(0.11)$, whereas 25.93 $\mathrm{mg} \cdot \mathrm{dm}^{-3} \mathrm{~N}$ and the lowest $\mathrm{P}$ dose caused the highest $\mathrm{P}$ recovery efficiency $(0.24)$, Figure 1(d).

$\mathrm{Ca}_{2}\left(\mathrm{H}_{2} \mathrm{PO}_{4}\right)$ contains $150 \mathrm{~g} \mathrm{Ca} \mathrm{kg}^{-1}$, therefore supplementation with crescent $\mathrm{P}$ doses resulted in increased Ca levels, and consequently higher sum of bases, base saturation and CEC. The soil acidification (characterized by a $\mathrm{pH}$ varying between 5.71 and 6.43) resulting from elevated $\mathrm{H}+\mathrm{Al}$ as a consequence of $\mathrm{N}$ addition increased $\mathrm{P}$ availability in the soil solution [26]. 


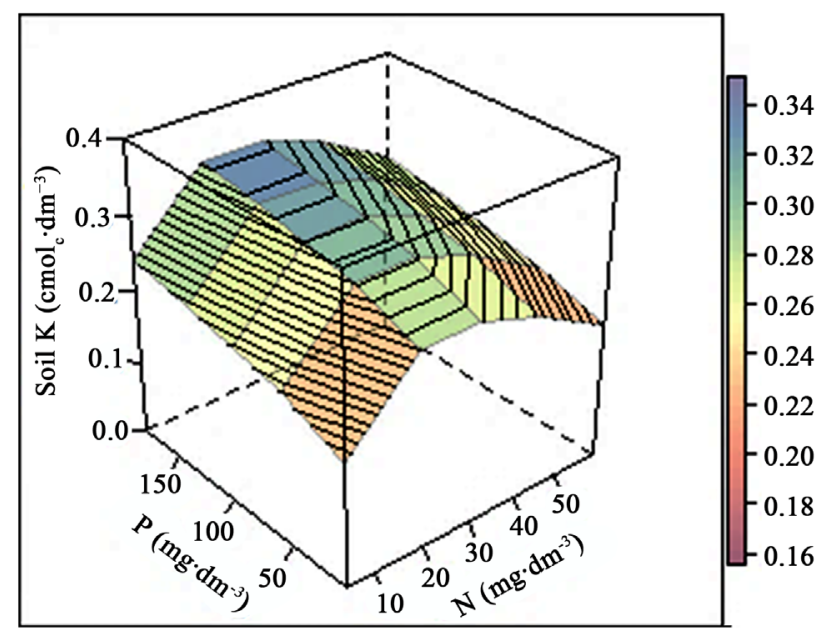

(a)

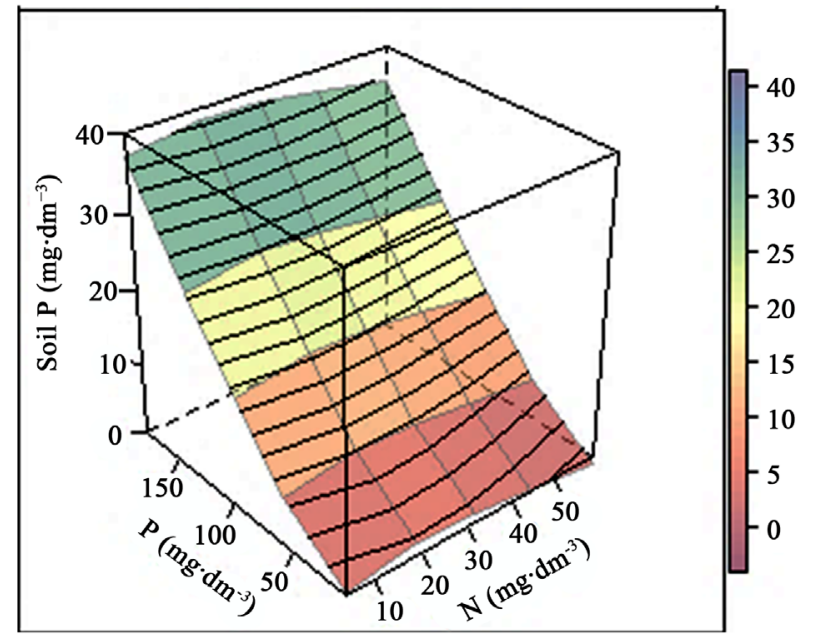

(c)

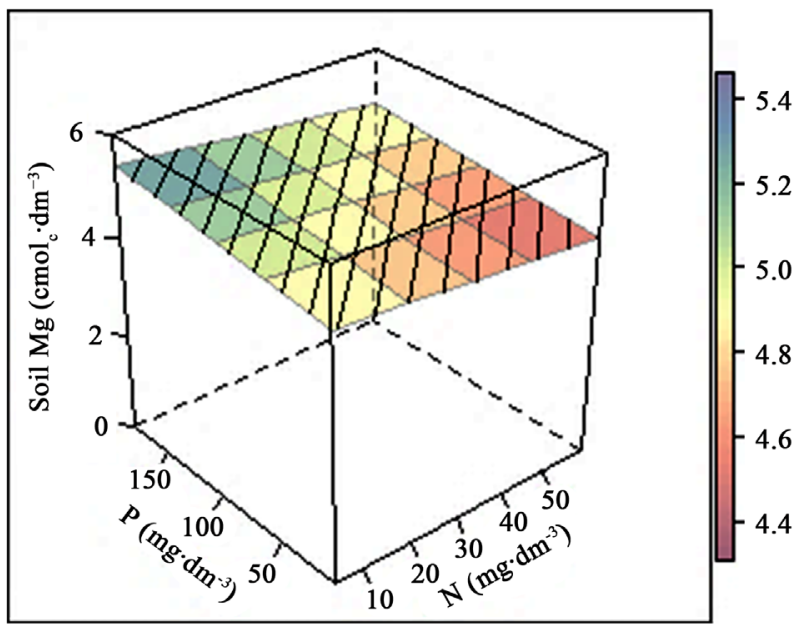

(b)

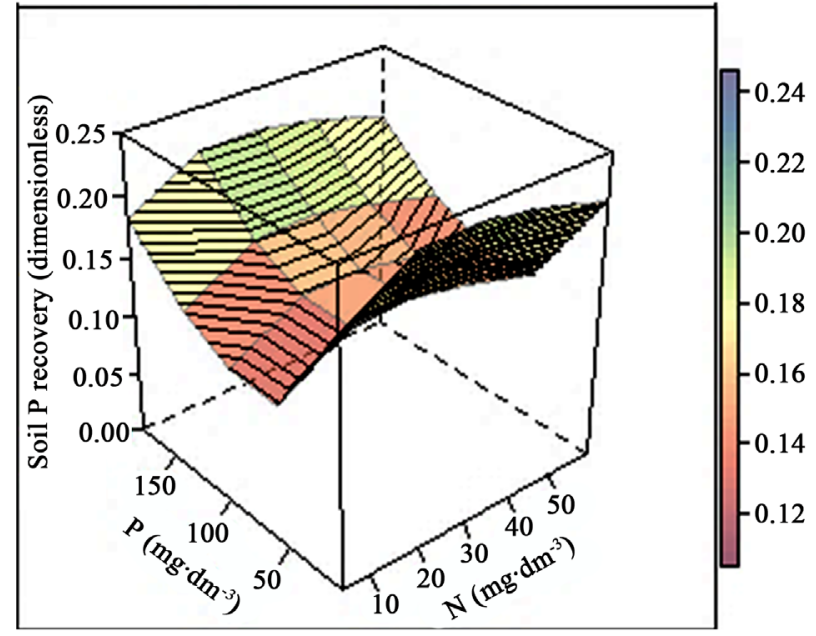

(d)

Figure 1. Effect of nitrogen (N) and phosphorus (P) fertilization on soil chemistry. K (a), Mg (b), P (c) and expected P/soil P (d) by nitrogen and phosphorus doses. Fitted models are in Table 1. Soil sampled at 259 DAT, sample composed of substrate collected from two vases. $\mathrm{Mg}(\mathrm{KCl}), \mathrm{P}$ and $\mathrm{K}$ (Mehlich 1).

\subsection{Effect of Plant Age or Age at Harvest and of $N$ and $P$ Fertilization on the Carobinha Development}

Plant age had an effect on plant height and stem diameter $(p<0.01)$, while age at harvest affected the number of branches, xylopodium diameter, leaf number, and leaf area $(p<0.01)$. Plant height slowly increased up to 105 DAT $(0.0000059$ $\left.-0.0012 \mathrm{~cm} \cdot \mathrm{day}^{-1}\right)$, and then rapidly until 359 DAT $\left(0.0024-0.1408 \mathrm{~cm} \cdot \mathrm{day}^{-1}\right)$, with a peak at 200 DAT. After that, the growth rate decreased from 0.00079 to $0.000011 \mathrm{~cm} \cdot \mathrm{day}^{-1}$ at $662 \mathrm{DAT}$ (Figure 2(a1)). Likewise, stem diameter slowly increased until 121 DAT $\left(0.0000099-0.0024 \mathrm{~mm} \cdot\right.$ day $\left.^{-1}\right)$, rapidly until 426 DAT (0.0021 - $\left.0.0665 \mathrm{~mm} \cdot \mathrm{day}^{-1}\right)$, reaching a peak at $218 \mathrm{DAT}$, and continued growing at a reduced rate (from 0.00087 to $0.000018 \mathrm{~mm} \cdot \mathrm{day}^{-1}$ ) until $672 \mathrm{DAT}$ (Figure 2(b1)). From 259 DAT, stem diameter increased at a higher rate [up to $372.04 \%$ $(\mathrm{cm} / \mathrm{mm})$ higher at $662 \mathrm{DAT}]$ than plant height. 
Supplementation with both $\mathrm{N}$ and $\mathrm{P}$ resulted in an elevation in plant height ( $p$ $=0.01)$. Increasing the $\mathrm{N}$ dose up to $26.03 \mathrm{mg} \cdot \mathrm{dm}^{-3}$ and the $\mathrm{P}$ dose up to 109.23 $\mathrm{mg} \cdot \mathrm{dm}^{-3}$ led the plants to reach their maximum height $(9.90 \mathrm{~cm})$ (Figure 2(a2)). $P$ addition also increased stem diameter in up to $54.42 \%(p<0.01$ and $p=0.01)$ at the highest dose (Figure 2(b2)).

Between 259 and 770 DAT, the number of branches expanded $1518.22 \%$ (Figure 3(a)), and, xylopodium diameter, 66.49\% (Figure 3(b)). However, the leaf area reduced $87.43 \%$ (Figure $3(c)$ ).

$\mathrm{N}$ slightly promoted branching $(p=0.07)$ (Figure $3(\mathrm{a})$ ) and acted inversely on xylopodium diameter $(p=0.01)$ (Figure $3(\mathrm{~b}))$ and leaf area $(p=0.04)$ (Figure $3(c)$ ) between 259 and 770 DAT. $N$ addition led to the maximum branching (4.65 branches/plant at $259 \mathrm{DAT}$ and 7.94 branches/plant at $770 \mathrm{DAT}$ ) at the highest dose (minimal P) (Figure 3(a)). At 259 DAT, the application of intermediate doses of $\mathrm{N}$ led to a reduction in xylopodium diameter until the lowest value of $8.61 \mathrm{~mm} /$ plant at a dose of $19.79 \mathrm{mg} \cdot \mathrm{dm}^{-3} \mathrm{~N}$ (Figure 3(b)) and the smallest leaf area of $328.65 \mathrm{~mm}^{2} / \mathrm{plant}$ at a dose of $26.30 \mathrm{mg} \cdot \mathrm{dm}^{-3} \mathrm{~N}$ (minimal P)

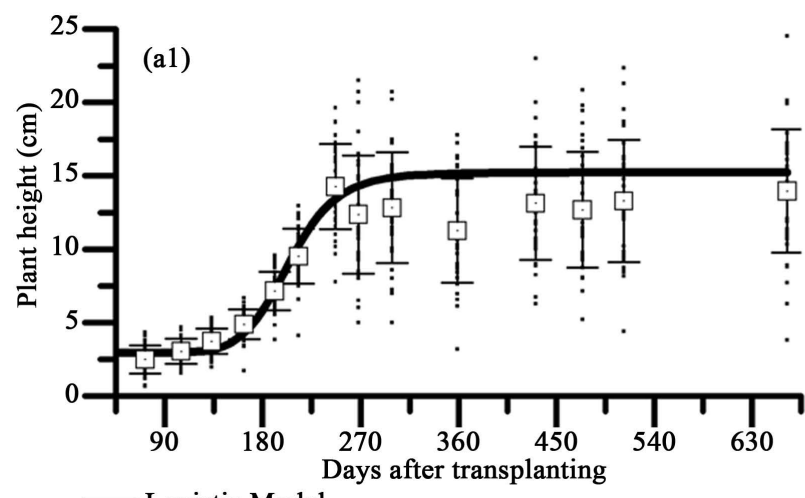

Logistic Model

- Plant height $(\mathrm{cm})$

$\square$ Mean

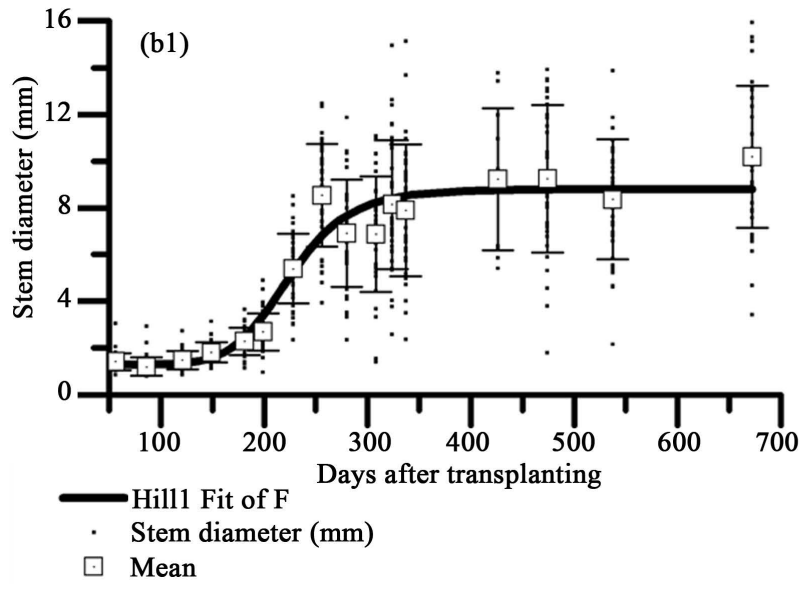

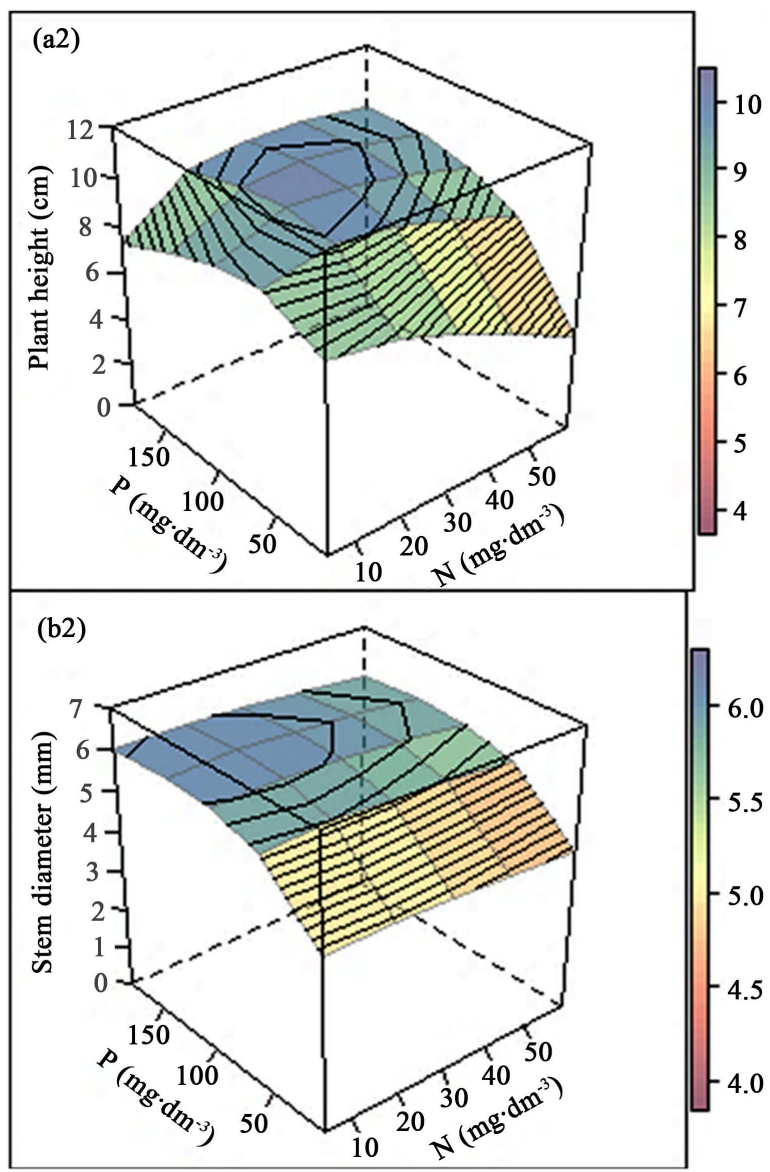

Figure 2. Effects of plant age or age at harvest and of nitrogen $(\mathrm{N})$ and phosphorus $(\mathrm{P})$ fertilization on the carobinha growth. Plant height ((a) and (b)) and stem diameter ((c) and (d)) by plant age (1) $(n=72-144)$ and the $\mathrm{N}$ and P doses (2) ( $n=2-4)$. Fitted models are in Table 1. Plant height and stem diameter refer to the average values between treatments. Bars indicate standard error $( \pm)$. 


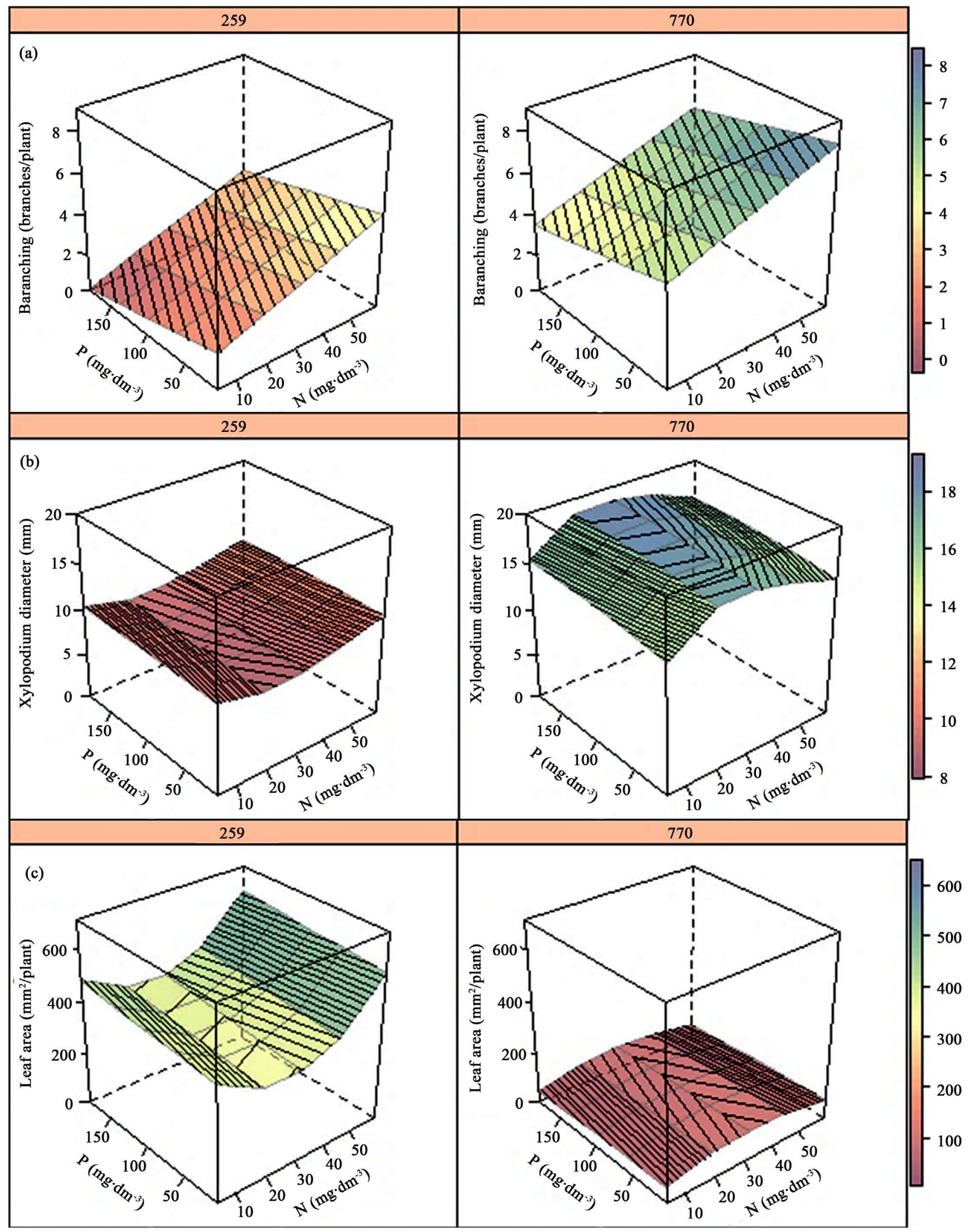

Figure 3. Effects of plant age or age at harvest and of nitrogen $(\mathrm{N})$ and phosphorus $(\mathrm{P})$ fertilization on the carobinha growth. Branching (a), xylopodium diameter (b) and leaf area (c) by age at harvest and the $\mathrm{N}$ and $\mathrm{P}$ doses $(n=2)$. Fitted models are in Table 1. 
(Figure 3(c)). However, at $770 \mathrm{DAT}$, the addition of intermediate $\mathrm{N}$ doses caused the xylopodium to reach its largest diameter $(18.72 \mathrm{~mm} / \mathrm{plant})$ at 25.69 $\mathrm{mg} \cdot \mathrm{dm}^{-3}$ of N (highest P) and leaf area $\left(108.02 \mathrm{~mm}^{2} /\right.$ plant) at $33.47 \mathrm{mg} \cdot \mathrm{dm}^{-3}$ of $\mathrm{N}$ (lowest P).

Plants at 259 DAT produced more leaves (Figure 3(c) and Figure 4(a)). In contrast, the plants produced less xylopodium (Figure 4(b)) and dry roots (Figure $4(\mathrm{~d}))(\mathrm{p}<0.01)$ than those at 770 DAT. Plants at 770 DAT produced $90.99 \%$ less dry leaves, $261.83 \%$ more dry xylopodium and $121.57 \%$ more dry roots. Applying N increased production of dry leaves $(p=0.04)$ (Figure 4(a)) at 259 DAT. In addition, the $\mathrm{N}$ promoted the production of dry xylopodium ( $\mathrm{p}<$ 0.10 ) (Figure $4(\mathrm{~b})$ ). Supplementation with $P$ caused a higher production of dry stem (Figure $4(\mathrm{c})$ ). At 259 DAT harvest, when applied $\mathrm{N}$ dose of $21.71 \mathrm{mg} \cdot \mathrm{dm}^{-3}$ plants produced the lowest dry leaves biomass (6.08 g/plant) (highest P). However, at highest $\mathrm{N}$ dose the plants produced the highest dry leaves biomass (12.22 $\mathrm{g} /$ plant) (minimal P). In regard with plants at $770 \mathrm{DAT}$, the addition of $\mathrm{N}$ doses up to $38.85 \mathrm{mg} \cdot \mathrm{dm}^{-3}$ increased dry leaf production until a maximum of 1.15 $\mathrm{g} /$ plant (minimal P). Supplementation with P led to the largest increase in the production of dry stem $\left(3.15 \mathrm{~g} /\right.$ plant at $\left.84.39 \mathrm{mg} \cdot \mathrm{dm}^{-3}\right)$ (minimal $\left.\mathrm{N}\right)$. $\mathrm{N}$ addition resulted in xylopodium production growing proportionally to the fertilization dose: the highest dose caused the highest production of dry xylopodium (2.49 g/plant at 259 DAT and $10.16 \mathrm{~g} /$ plant at 770 DAT) (minimal P).

The growth of the carobinha is age and season dependent. As the life cycle progresses, more stem and more branches are produced in detriment of height, with a reflection on the increased growth of the xylopodium, a storage organ important for the regrowth after the dormancy period and environmental stresses [27]. This phenomenon defines the change in the growth behavior along the life cycle, justifying the formation of underground forests in native areas, with sprouts emerging from the xylopodium or from the stem base [28]. The large decrease in leaf number in the older plants indicates that the deciduousness typical of the species [29] occurs even in the absence of a water deficit. Considering that the carobinha organs used in the traditional medicine are the leaves and the roots [5] [6], the best time to harvest must be taken into account, since, the older the plant, the higher root production is. On the other hand, leaf production is larger upon regrowth in summer and lower in winter.

Within the fertilizer levels tested, some traits of plant growth (plant height and stem diameter) reached their highest values for $\mathrm{N}$ and $\mathrm{P}$ as demonstrated by [17] for Acer mono. However, several traits reached their highest values only for $\mathrm{N}$ levels (diameter of xylopodium, leaf area and dry leaves biomass) or P levels (fresh stem) and others (dry branches and xylopodium biomass) did not reach their highest growth within the doses of $\mathrm{N}$ and $\mathrm{P}$ tested. At the beginning $\mathrm{N}$ probably promoted stem cellular division, since it constitutes the aminoacids required in large amount in the initial growth stages. It later promoted cell elongation and stem development [30]. Larger leaf area during physiological dormancy may increase water loss, causing a hydric unbalance in the plants. The 

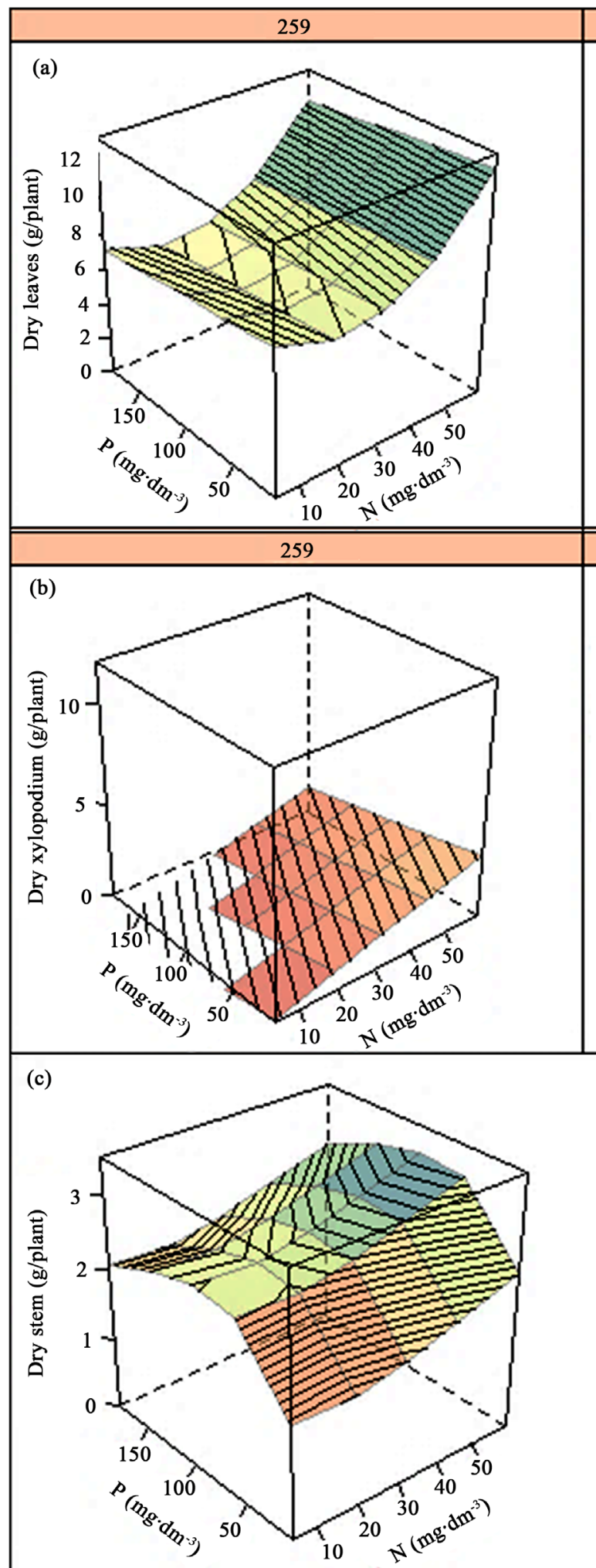
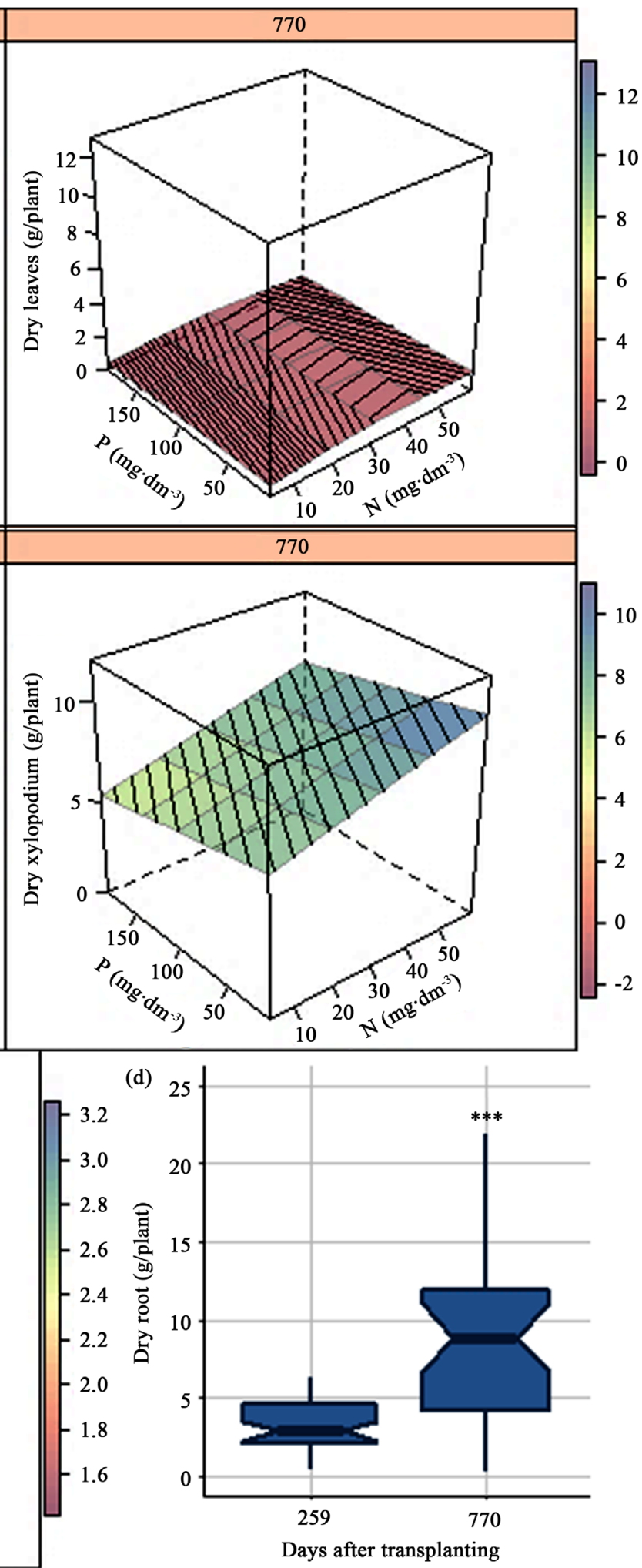

Figure 4. Effects of age at harvest and of nitrogen $(\mathrm{N})$ and phosphorus $(\mathrm{P})$ fertilization on carobinha yield. Production of dries leaves (a); dry xylopodium (b) by age at harvest and $\mathrm{N}$ and P doses $(n=2)$. Dry stem (c) by $\mathrm{N}$ and P doses $(\mathrm{n}=4)$. Dry root $(\mathrm{d})$ by age at harvest $(n=36)$. Fitted models are in Table 1 . Stem production is the average of both harvests $(259$ and 770 DAT), and root production refers to the average between treatments. Bars in the boxplot graphs indicate variability outside the upper and lower quartile, out-of-graph points indicate outliers, and the width of the box indicates the sample size. ${ }^{* *} p>0.001$ by $\mathrm{F}$ test. 
fact that fertilization with nitrogen and phosphorus had no effect on root production seems to confirm that root growth does not depend on nutrient supplementation [29] [31] [32]. However, in a previous study, the addition of P together with chicken manure fertilizer led to increased root production [13]. In the present work, we showed that nitrogen fertilization affects the development of the aerial part in the carobinha, reflected in a higher number of leaves, whereas supplementation with $\mathrm{P}$ increases stem production, an interesting observation considering the change in plant growth.

\subsection{Effect of Age at Harvest and $\mathbf{N}$ and $P$ Fertilization on Nutrient Uptake}

Macronutrient concentration decrease in older plants. In the leaves between 259 and $770 \mathrm{DAT}$, Ca levels reduced 14.23\% $(p=0.03)$ (Figure 5(a)); Mg levels $39.40 \%(p<0.01)$ (Figure 5(b)) and $\mathrm{N}$ levels were $35.01 \%$ lower $(p<0.01)$ (Figure 5(c1)). Soil fertilization with $\mathrm{P}$ affected the concentration of $\mathrm{K}(p=$ $0.04), \mathrm{N}(p<0.07)$ and $\mathrm{P}(p=0.01)$ in the leaves as well; however, $\mathrm{N}$ and $\mathrm{P}$ had

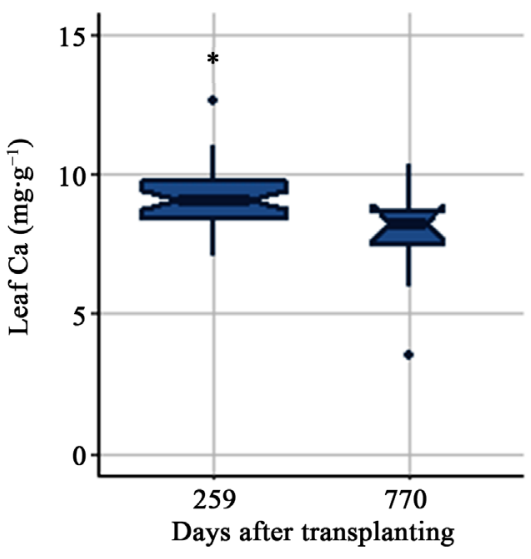

(a)

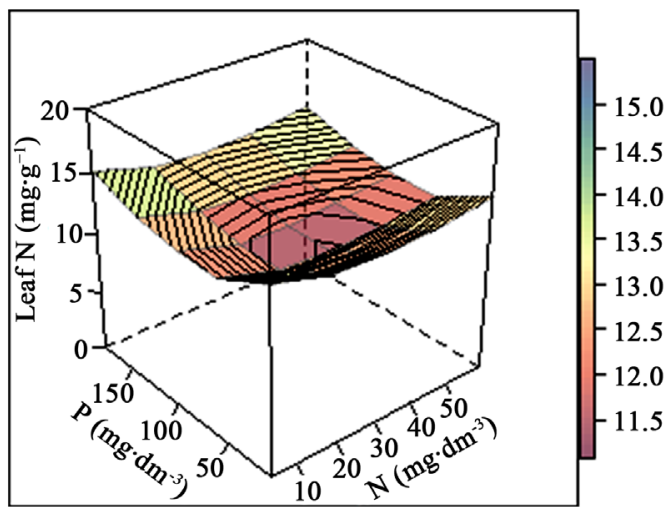

(c2)

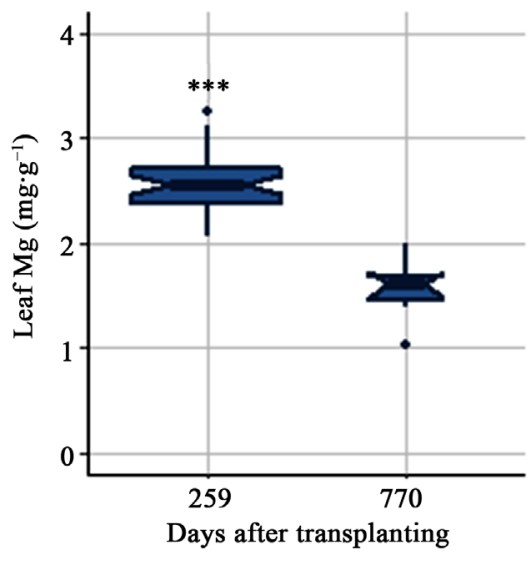

(b)

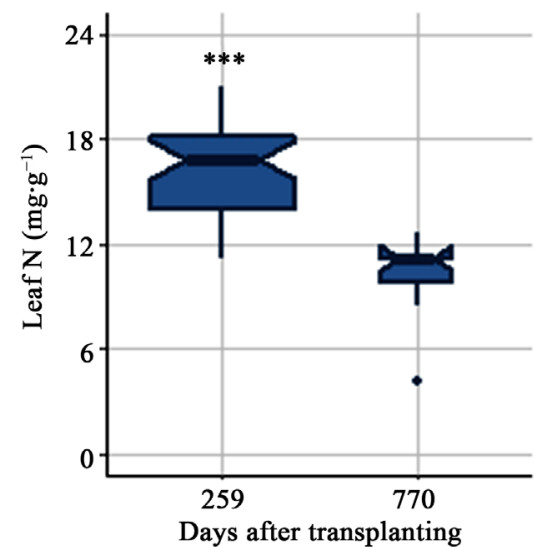

(c1)

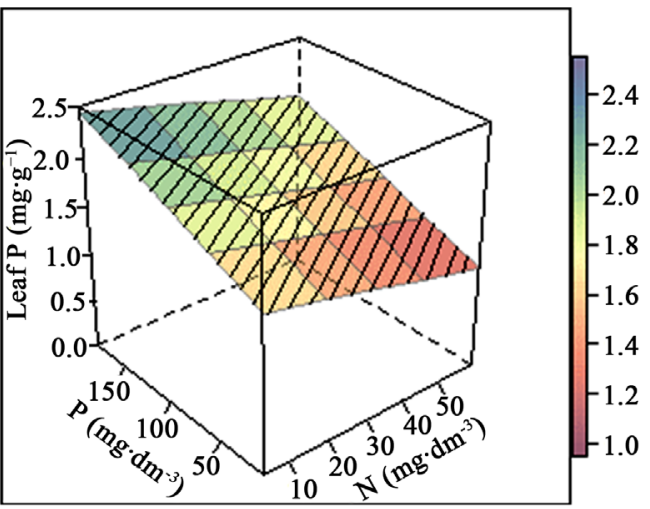

(d)

Figure 5. Effects of age at harvest and of nitrogen $(\mathrm{N})$ and phosphorus $(\mathrm{P})$ fertilization on macronutrient concentrations in carobinha leaves. $\mathrm{Ca}(\mathrm{a}), \mathrm{Mg}(\mathrm{b})$ and $\mathrm{N}(\mathrm{c} 1)$ by age at harvest $(n=36) . \mathrm{N}(\mathrm{c} 2)$ and $\mathrm{P}(\mathrm{d})$ by $\mathrm{N}$ and $\mathrm{P}$ doses in the plants harvested at 259 DAT $(n=8)$. Fitted models are in Table $1 . \mathrm{Ca}, \mathrm{Mg}$ and $\mathrm{N}$ levels represent the average of treatments. Bars in the boxplot graphs indicate variability outside the upper and lower quartile, out-of-graph points indicate outliers, and the width of the box indicates the sample size. ${ }^{\star} p>0.05,{ }^{* *} p>0.01,{ }^{* * *} p>0.001$, and ${ }^{\star * * *} p>0.0001$ by F test. 
no effect on $\mathrm{Ca}(\mathrm{N}, p=0.98$ and $\mathrm{P}, p=0.80)$ and $\mathrm{Mg}$ levels $(\mathrm{N}, p=0.85$ e $\mathrm{P}, p=$ $0.59)$ in the leaves. Regarding $\mathrm{K}$, the $\mathrm{P}$ coefficients were significant in the quadratic $(p=0.04)$ and quadratic root $(p=0.02)$ models, while the coefficients of regression determination were not $\left(\mathrm{R}^{2}=0.22, p=0.21\right.$ and $\mathrm{R}^{2}=0.26, p>0.10$, respectively). Supplementation with $\mathrm{P}$ reduces the $\mathrm{N}$ levels from highest (15.20 $\left.\mathrm{mg} \cdot \mathrm{g}^{-1}\right)$ at the minimal $\mathrm{P}$ dose to lowest $\left(12.06 \mathrm{mg} \cdot \mathrm{g}^{-1}\right)$ at a dose of 69.31 $\mathrm{mg} \cdot \mathrm{dm}^{-3}$ of $\mathrm{P}$ (highest $\mathrm{N}$ ) (Figure $5(\mathrm{c} 2)$ ). Nevertheless, the addition of $\mathrm{P}$ proportionally increased the $\mathrm{P}$ concentrations, reaching a maximum of $1.65 \mathrm{mg} \cdot \mathrm{g}^{-1}$ at the highest dose (minimal N) (Figure 5(d)).

In the roots, between 259 and $770 \mathrm{DAT}$, K levels decreased $50.32 \%(p<0.01)$ (Figure 6(a)); Ca levels 7.08\% ( $p=0.04$ ) (Figure 6(b)); Mg levels 29.80\% ( $p<$ 0.01 ) (Figure 6(c)) and $\mathrm{N}$ levels were 59.91\% lower $(p<0.01)$ (Figure 6(d)). $\mathrm{P}$ concentration constituted an exception, increasing 232.74\% $(p<0.01)$ (Figure $6(\mathrm{e}))$.

Addition of $\mathrm{N}$ and $\mathrm{P}$ did not affect $\mathrm{K}(\mathrm{N}, p=0.36$ and $\mathrm{P}, p=0.46)$ or Ca levels $(\mathrm{N}, p=0.27$ and $\mathrm{P}, p=0.81)$ on the roots. However, adding $\mathrm{N}$ increased $\mathrm{Mg}$ levels at $259 \mathrm{DAT}$ and reduced Mg levels at $770 \mathrm{DAT}(p=0.03)$. Fertilization with $\mathrm{P}$ led to increased $\mathrm{Mg}$ levels at both harvests $(p<0.05)$ (Figure 6(c)). At 259 DAT, $30.03 \mathrm{mg} \cdot \mathrm{dm}^{-3} \mathrm{~N}$ and the highest $\mathrm{P}$ dose resulted in the highest Mg levels $\left(2.84 \mathrm{mg} \cdot \mathrm{g}^{-1}\right)$, while, at $770 \mathrm{DAT}, 39.80 \mathrm{mg} \cdot \mathrm{dm}^{-3}$ of $\mathrm{N}$ and the minimal $\mathrm{P}$ dose resulted in the lowest $\mathrm{Mg}$ levels $\left(1.46 \mathrm{mg} \cdot \mathrm{g}^{-1}\right)$. Supplementation with both $\mathrm{N}(p=$ $0.01)$ and $\mathrm{P}(p=0.04)$ led to increased $\mathrm{N}$ concentrations (Figure 6(d)); and $43.32 \mathrm{mg} \cdot \mathrm{dm}^{-3} \mathrm{~N}$ and the higher $\mathrm{P}$ dose led to the maximum $\mathrm{N}$ concentrations (15.11 $\mathrm{mg} \mathrm{g}^{-1}$, at $259 \mathrm{DAT}$ and $7.43 \mathrm{mg} \cdot \mathrm{g}^{-1}$ at $\left.770 \mathrm{DAT}\right)$. However, $\mathrm{P}$ acted more expressively at the dose of $133.86 \mathrm{mg} \cdot \mathrm{dm}^{-3}$ or higher. In this context, the minimal $\mathrm{N}$ dose and $73.70 \mathrm{mg} \cdot \mathrm{dm}^{-3} \mathrm{P}$ resulted in the lowest $\mathrm{N}$ levels $\left(9.76 \mathrm{mg} \cdot \mathrm{g}^{-1}\right.$ at 259 DAT and $2.08 \mathrm{mg} \cdot \mathrm{g}^{-1}$ at $\left.770 \mathrm{DAT}\right)$. The combined addition of $\mathrm{N}$ and $\mathrm{P}$ led to increased P concentrations at 259 DAT (Figure 6(e)); the addition of 49.97 $\mathrm{mg} \cdot \mathrm{dm}^{-3}$ of $\mathrm{N}$ and the maximum $\mathrm{P}$ dose increased the $\mathrm{P}$ concentration to its maximum $\left(3.05 \mathrm{mg} \cdot \mathrm{g}^{-1}\right)$. However, at $770 \mathrm{DAT}$ the isolated effect of each nutrient was stronger than their combined action. At the lowest $\mathrm{P}$ dose, $\mathrm{N}$ addition elevated $\mathrm{P}$ concentration from $1.04 \mathrm{mg} \cdot \mathrm{g}^{-1}\left(4.20 \mathrm{mg} \cdot \mathrm{dm}^{-3}\right)$ to $2.97 \mathrm{mg} \mathrm{g}^{-1}(59.85$ $\left.\mathrm{mg} \cdot \mathrm{dm}^{-3}\right)(p=0.04)$.

In the leaves, age at harvest and $\mathrm{N}$ fertilization had isolated effects on content levels of $\mathrm{K}$ (harvest, $p<0.01$ and $\mathrm{N}, p=0.03$ ) (Figure 7(a)) and Ca (harvest, $p<$ 0.01 and $\mathrm{N}, p=0.06$ ) (Figure $7(\mathrm{~b})$ ). Combined, they had a slight effect on $\mathrm{Mg}$ contents $(p=0.09)$ (Figure $7(\mathrm{c}))$. Age at harvest isolated, together with $\mathrm{N}$ and with $\mathrm{P}$ addition acted on $\mathrm{N}$ contents (harvest, $p<0.01, \mathrm{~N}^{2 \star}$ harvest, $p=0.09$, and $\mathrm{P}^{2 *}$ harvest, $p=0.07$ ) (Figure $7(\mathrm{~d})$ ). Age at harvest isolated, together with $\mathrm{P}$, and $\mathrm{N}$ isolated addition acted on $\mathrm{P}$ contents (harvest, $p<0.01, \mathrm{P}^{\star}$ harvest, $p=0.09$, and $\mathrm{N}, p=0.09$ ) (Figure $7(\mathrm{e})$ ). The increase in plants age resulted in a decrease of $85.90 \%$ in $\mathrm{K}$, of $79.33 \%$ in $\mathrm{Ca}$, of $88.92 \%$ in $\mathrm{N}$ and of $90.26 \%$ in P levels. Increasing amounts of $\mathrm{N}$ elevated proportionally the contents of $\mathrm{K}, \mathrm{Ca}$ and $\mathrm{P}$. The 


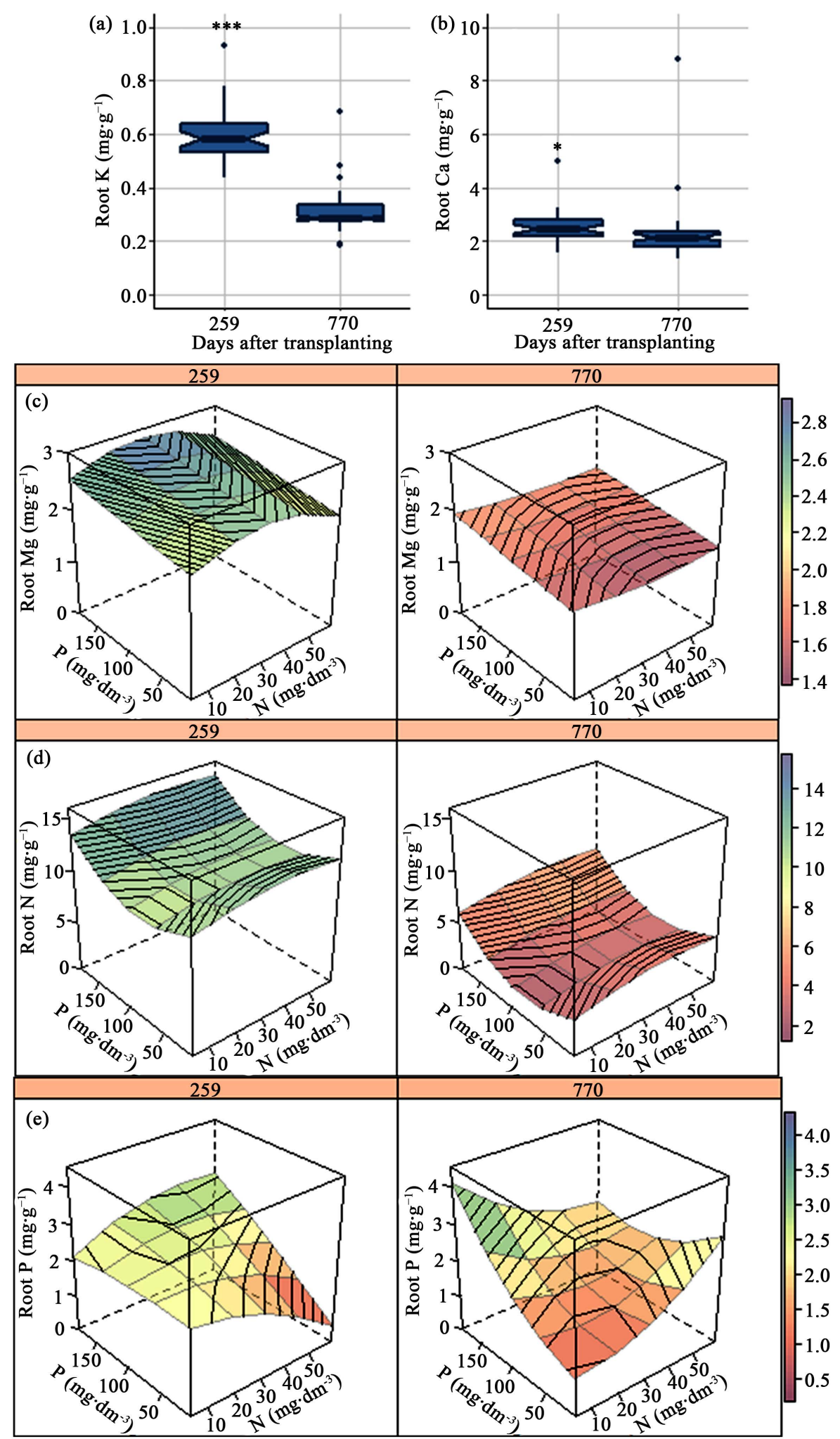

Figure 6. Effects of age at harvest and of nitrogen $(\mathrm{N})$ and phosphorus $(\mathrm{P})$ fertilization on macronutrient concentrations in carobinha roots. $\mathrm{K}(\mathrm{a})$ and $\mathrm{Ca}(\mathrm{b})$ by age at harvest $(n=36) . \mathrm{Mg}(\mathrm{c}), \mathrm{N}(\mathrm{d})$ and $\mathrm{P}(\mathrm{e})$ by age at harvest and $\mathrm{N}$ and $\mathrm{P}$ doses $(n=8)$. Fitted models are in Table $1 . \mathrm{K}$ and Ca represent the average of treatments. Bars in the boxplot graphs indicate variability outside the upper and lower quartile, out-of-graph points indicate outliers, and the width of the box indicates the sample size. ${ }^{\star} p>0.05,{ }^{\star *} p>$ $0.01,{ }^{\star * \star} p>0.001$, and ${ }^{\star * \star *} p>0.0001$ by $\mathrm{F}$ test. 


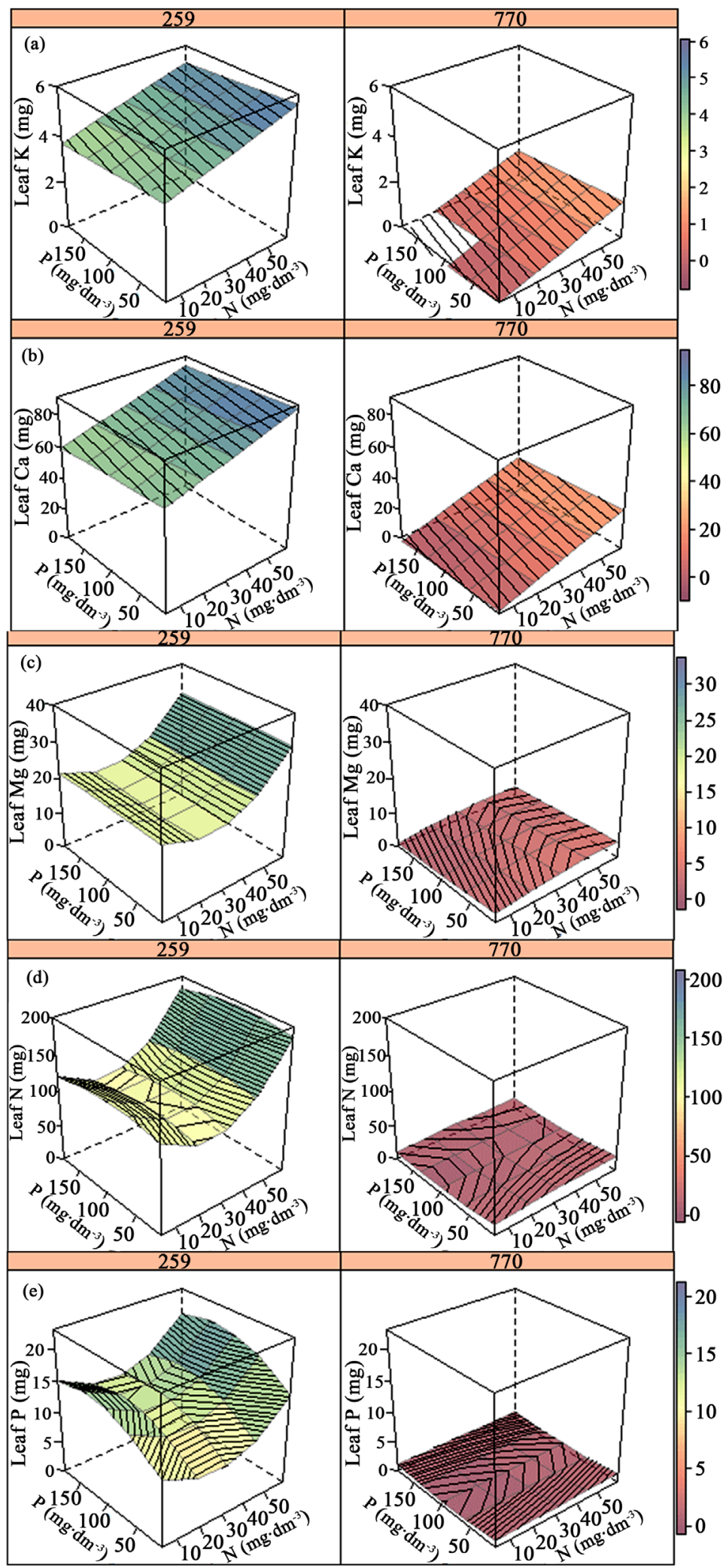

Figure 7. Effects of age at harvest and of nitrogen $(\mathrm{N})$ and phosphorus $(\mathrm{P})$ fertilization on nutrient contents in the carobinha. Contents of $\mathrm{K}(\mathrm{a}), \mathrm{Ca}(\mathrm{b}), \mathrm{Mg}(\mathrm{c}), \mathrm{N}(\mathrm{d})$ and $\mathrm{P}(\mathrm{e})$ in the leaves by age at harvest $(259, n=8$ and $770 \mathrm{DAT}, n=5)$ and $\mathrm{N}$ and P doses. Fitted models are in Table 1. 
highest $\mathrm{N}$ dose led to the highest $\mathrm{K}(5.63 \mathrm{mg}$ at $259 \mathrm{DAT}$ and $1.61 \mathrm{mg}$ at 770 $\mathrm{DAT}), \mathrm{Ca}(88.23 \mathrm{mg}$ at $259 \mathrm{DAT}$ and $26.19 \mathrm{mg}$ at $770 \mathrm{DAT})$. The highest $\mathrm{N}$ dose regardless of harvest ages led to the highest leaf P contents $(19.86 \mathrm{mg}$ at 259 DAT and $1.51 \mathrm{mg}$ at $770 \mathrm{DAT}$ ) but with an increase in the P dose up to 129.33 $\mathrm{mg} \cdot \mathrm{dm}^{-3}$ in the younger plants (259 DAT) and to the highest $\mathrm{P}$ dose in the older plants (770 DAT). On the other hand, in the older plants, an increase in the $\mathrm{N}$ dose up to $47.24 \mathrm{mg} \cdot \mathrm{dm}^{-3}$ elevated the $\mathrm{Mg}$ levels to a maximum of $23.72 \mathrm{mg}$ (minimal P). An increment in the P dose up to $90 \mathrm{mg} \cdot \mathrm{dm}^{-3}$ at $259 \mathrm{DAT}$ and the minimum $\mathrm{P}$ dose at $770 \mathrm{DAT}$, both with maximum $\mathrm{N}$ dose increased the leaf $\mathrm{N}$ content to the highest levels (193.85 mg at $259 \mathrm{DAT}$ and $5.22 \mathrm{mg}$ at $770 \mathrm{DAT}$ ).

In the roots, age at harvest and $\mathrm{N}(p>0.99)$ and $\mathrm{P}(p=0.81)$ addition did not affect $\mathrm{K}$ contents. However, age at harvest and $\mathrm{P}$ addition had a slight effect on Ca contents $p=0.08$ ) (Figure 8(a)). In the plants at $259 \mathrm{DAT}$, an increment in the $\mathrm{P}$ dose up to $62.52 \mathrm{mg} \cdot \mathrm{dm}^{-3}$ resulted in the highest levels of radicular $\mathrm{Ca}$ $(17.19 \mathrm{mg})$, while an increase to the maximum $\mathrm{P}$ dose resulted in the lowest $\mathrm{Ca}$ contents $(10.72 \mathrm{mg})$. As the plants aged, Ca contents decreased, whereas P addition starting at a dose of $47.82 \mathrm{mg} \cdot \mathrm{dm}^{-3}$ increased these levels from a minimum of $18.34 \mathrm{mg}$ until a maximum of $45.18 \mathrm{mg}$ at the highest $\mathrm{P}$ dose. On the other hand, with aging, $\mathrm{Mg}$ contents increased 69.57\% $(p=0.01)(p=0.01)$ (Figure $8(\mathrm{~b}))$. The $\mathrm{N}$ addition affected $\mathrm{N}$ contents in the roots $(p=0.02)$ (Figure 8(c)). However, the linear $\left(\mathrm{R}^{2}=0.108, p=0.19\right)$ and quadratic root models $\left(\mathrm{R}^{2}=0.101\right.$, $p=0.23$ ) were not adjusted to the effects of $\mathrm{N}$. The $\mathrm{N}$ addition proportionally increased the levels of radicular $\mathrm{N}$ up to a maximum of $58.09 \mathrm{mg}$ at the highest $\mathrm{N}$ dose. Together with age at harvest, $\mathrm{N}$ and $\mathrm{P}$ addition affected $\mathrm{P}$ contents $(p=$ 0.04) (Figure 8(d)). In the plants at $259 \mathrm{DAT}$, the highest $\mathrm{N}$ and $\mathrm{P}$ doses led to the highest $\mathrm{P}$ levels $(12.90 \mathrm{mg})$. However, the same $\mathrm{N}$ dose combined with the lowest $\mathrm{P}$ dose reduced the $\mathrm{P}$ contents to the minimum $(2.94 \mathrm{mg})$. In the older plants (770 DAT), the lower $\mathrm{N}$ dose combined with the higher $\mathrm{P}$ dose resulted in a higher P level $(55.63 \mathrm{mg}$ ), while the same $\mathrm{N}$ dose together with the lower $\mathrm{P}$ dose led a lower P content $(9.45 \mathrm{mg})$.

The effect of fertilization or other treatments on nutrient uptake in the plant tissues depends on the combined action of each of them on the nutrient concentration and contents in the plant and on biomass production [33]. When the carobinha is younger and the roots are smaller, there is $\mathrm{Ca}$ and $\mathrm{Mg}$ concentration in the roots and antagonism to P. However, as the plant ages, the root biomass increases, therefore causing a dilution of radicular $\mathrm{Ca}$ and $\mathrm{Mg}$ and $\mathrm{P}$ synergism in the roots. This occurs because, upon growing, the roots reach the less-exchangeable $\mathrm{P}$ in the soil due to increased radicular interception and a lower diffusion distance, incrementing nutrient uptake [11] [34]. Leaf deciduousness in older plants (770 DAT) may have led to $\mathrm{Mg}$ and $\mathrm{N}$ translocation from older to younger leaves. However, regarding the levels of $\mathrm{Ca}$, which does not move in the leaves, age progression led to a two-fold reduction in the leaves when compared to the reduction observed in the roots (Figure 7 (b) and Figure $8(a)$ ), indicating that Ca translocation is very low or null. In the younger plants, 

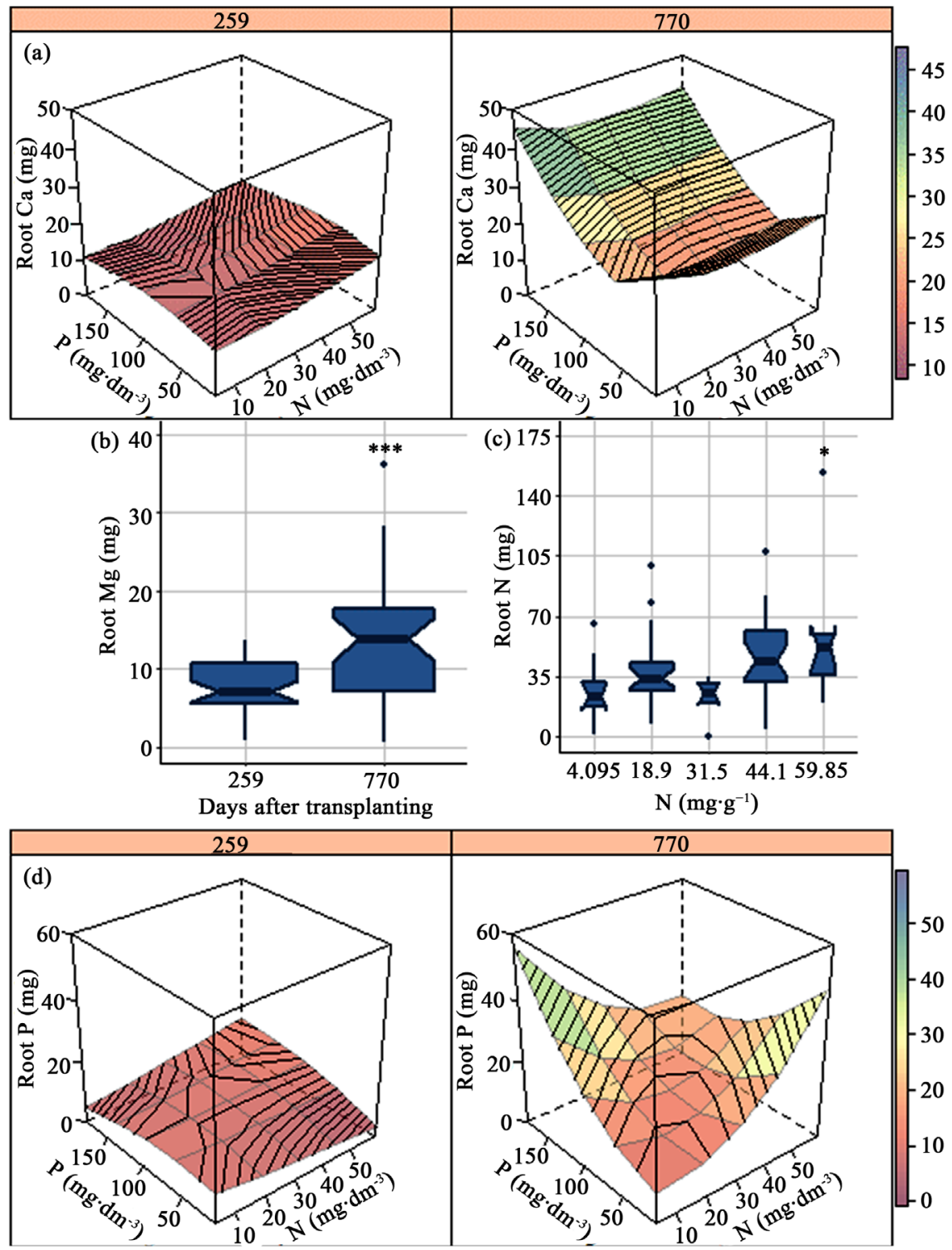

Figure 8. Effects of age at harvest and of nitrogen $(\mathrm{N})$ and phosphorus $(\mathrm{P})$ fertilization on nutrient contents in the carobinha roots. Contents of $\mathrm{Ca}(\mathrm{a})$ and $\mathrm{P}(\mathrm{d})$ by harvest age (259, $n=8$ and 770 DAT, $n=5$ ) and, N and P doses. Mg contents (b) by plant age at harvest $(259, n=72$ and $770 \mathrm{DAT}, n=45)$. $\mathrm{N}$ levels (c) by $\mathrm{N}$ doses $(n=16-48)$. Fitted models are in Table 1 . The Mg levels represents the average between treatments, and $\mathrm{N}$ contents refer to the average harvest age and the average $\mathrm{P}$ doses. Bars in the boxplot graphs indicate variability outside the upper and lower quartile, out-of-graph points indicate outliers, and the width of the box indicates the sample size. ${ }^{*} p>0.05,{ }^{* *} p>0.01$, ${ }^{* * *} p>0.001$, and ${ }^{* * * *} p>0.0001$ by $\mathrm{F}$ test.

$\mathrm{N}$ and $\mathrm{P}$ act synergistically on radicular $\mathrm{P}$ contents. On the other hand, in the older plants, the combined increase in the $\mathrm{N}$ and $\mathrm{P}$ doses had an antagonist effect on the levels of radicular P. Increased $\mathrm{N}$ availability usually leads to incremented P uptake [14]. Nevertheless, in the present work such effect occurred only in the younger plants. As they aged, the effect of $\mathrm{P}$ from the roots seemed to be more important than its synergism with $\mathrm{N}$. 
In the roots, supplementation with $\mathrm{N}$ acted synergistically with $\mathrm{N}$, regardless of age, and with $\mathrm{K}, \mathrm{Ca}, \mathrm{Mg}, \mathrm{N}$ and $\mathrm{P}$ in the leaves. However, it had no effect on $\mathrm{K}$ and $\mathrm{Ca}$ contents in the roots, since it did not affect radicular development. The growing addition of $\mathrm{S}$ doses in the form of ammonium sulfate may have increased the availability of ferredoxin $\left(\mathrm{Fe}_{2} \mathrm{~S}_{2}\right)$ involved in the transformation of glutamine into glutamate following $\mathrm{N}$ uptake [15]. Furthermore, $\mathrm{S}$ is also present in cysteine and methionine, both crucial aminoacids in the assimilation of $\mathrm{N}$ into proteins, incrementing foliar $\mathrm{N}$ contents. Enhanced $\mathrm{N}$ uptake by the plants increases $\mathrm{K}$ demand in the tissues, and may result in $\mathrm{K}$ deficiency or dilution [14]. However, fertilization with $\mathrm{K}$ must have been sufficient to supply the increased demand caused by the foliar growth promoted by N. Due to its role in the incremented foliar growth, $\mathrm{N}$ also increased $\mathrm{Ca}$ demands in the leaves. Besides, with the elevated chlorophyll levels caused by higher $\mathrm{N}$ availability, the demand for Mg, a chlorophyll constituent [12], increased as well.

Despite the fact that supplementation with $\mathrm{P}$ increased $\mathrm{Mg}$ and $\mathrm{N}$ concentrations in the roots and $\mathrm{P}$ levels in the leaves, reducing $\mathrm{K}$ and $\mathrm{N}$ levels in the leaves. Nevertheless, $\mathrm{P}$ did not show a clear effect on these levels, since it did not affect $\mathrm{Mg}$ and $\mathrm{N}$ contents in the roots or $\mathrm{K}$ contents in the leaves, but increased foliar $\mathrm{N}$ contents. Furthermore, $\mathrm{K}, \mathrm{Ca}$ and $\mathrm{Mg}$ contents in the leaves did not show any response to $\mathrm{P}$ fertilization. In the roots and leaves, $\mathrm{P}$ acted synergistically only with itself, due to the increased $\mathrm{P}$ availability in the soil.

This study was done under moderately controlled conditions, so in the field the plant response may be different. But it shows that the phosphorus and nitrogen fertilizer together allow further development of carobinha plants. Population studies can better clarify the role of $\mathrm{N}$ and $\mathrm{P}$ fertilization in the genetic diversity of the species. In addition, we observed high diversity among the plants, since the species has not yet been selected. Thus, both individuals responsive and efficient in the use of nutrients should be selected for commercial production [35]. We did not determine the levels of nitrate $\left(\mathrm{N}-\mathrm{NO}_{3}\right)$ and nitrite $\left(\mathrm{N}-\mathrm{NO}_{2}\right)$ in plant tissue, $\mathrm{N}$ forms that may be toxic to humans [36]. Therefore, future studies can determine these levels in the tissues and indicate the level of nitrogen fertilization in the carobinha that is safe for human health [37]. Future studies may also quantify the loss levels of $\mathrm{N}$ and $\mathrm{P}$.

\section{Conclusions}

$\mathrm{N}$ and $\mathrm{P}$ fertilization promotes the aerial development of plant and a differential allocation of nutrients between the carobinha tissues. $\mathrm{N}$ acts on the development of the aerial part, depending on the plant age, and on the nutrient allocation in the leaves. The effect of $\mathrm{P}$ on the development and nutrient allocation in the roots are independent of plant age. The carobinha grows as part of the sub-shrubs in the Cerrado. The growth is rapid in the younger plants and slower in adult plants. The growth changes from vertical to horizontal through stem development and the production of branches. The deciduousness in carobinha 
plants is independent of water availability. Nutrient contents in foliar and radicular tissues are usually lower in the adult plant, with the exception of $\mathrm{P}$, which increases. In the leaves, macronutrient levels increase with age, compared with the roots. In the younger plants, there is a nutrient concentration in the roots and an allocation synergy in the leaves. However, in adult plants, nutrient contents dilute in the roots, while in the leaves, exchangeable nutrients concentrate and less motile nutrients suffer an antagonistic effect.

\section{Acknowledgements}

The authors thank the Foundation for Support to the Development of Teaching, Science and Technology of the Mato Grosso do Sul State-FUNDECT and the Coordination for the Improvement of Higher Education Personnel-CAPES for the financial support for the accomplishment and publication of the present work.

\section{Conflict of Interest}

There are no conflicts of interest in present study.

\section{References}

[1] Hopkin, M. (2004) Brazilian Savannah “Will Disappear by 2030.” Nature. https://doi.org/10.1038/news040719-6

[2] Klink, C.A. and Machado, R.B. (2005) Conservation of the Brazilian Cerrado. Conservation Biology, 19, 707-713. https://doi.org/10.1111/j.1523-1739.2005.00702.x

[3] Farias, R. and Proença, C. (2003) Jacaranda decurrens subsp. symmetrifoliolata (Bignoniaceae), Novo Táxon Para O Bioma Cerrado. Boletim do Herbarium Bradeanum, 11, 5-9.

[4] Rezende, A.V., Walter, B.M.T., Fagg, C.W., Fagg, J.M.F., Júnior, M.C. da S., Noqueira, P.E., de Mendonça, R.C. and Filgueiras, T. de S. (2008) Cerrado: Ecologia e Flora. In: Sano, S.M., de Almeida, S.P. and Ribeiro, J.F., Eds., 2nd ed., Embrapa Informação Tecnológica, Brasília.

[5] Casagrande, J.C., Macorini, L.F.B., Antunes, K.A., dos Santos, U.P., Campos, J.F., Dias-Júnior, N.M., Sangalli, A., Lima Cardoso, C.A., do Carmo Vieira, M., Rabelo, L.A., Paredes-Gamero, E.J., dos Santos, E.L. and de Picoli Souza, K. (2014) Antioxidant and Cytotoxic Activity of Hydroethanolic Extract from Jacaranda decurrens Leaves. PLoS ONE, 9, e112748.

https://doi.org/10.1371/journal.pone.0112748

[6] Santos, J.A., Arruda, A., Silva, M.A., Cardoso, C.A.L., Vieira, M.D.C., Kassuya, C.A.L. and Arena, A.C. (2012) Anti-Inflammatory Effects and Acute Toxicity of Hydroethanolic Extract of Jacaranda decurrens Roots in Adult Male Rats. Journal of ethnopharmacology, 144, 802-805. https://doi.org/10.1016/j.jep.2012.10.024

[7] Antunes, K.A., Baldivia, D. da S., da Rocha, P. dos S., Casagrande, J.C., Argandoña, E.J.S., Vieira, M. do C., Cardoso, C.A.L., dos Santos, E.L. and de Picoli Souza, K. (2016) Antiobesity Effects of Hydroethanolic Extract of Jacaranda decurrens Leaves. Evidence-Based Complementary and Alternative Medicine, 2016, 1-8. https://doi.org/10.1155/2016/4353604

[8] Lannes, L.S., Bustamante, M.M.C., Edwards, P.J. and Olde Venterink, H. (2016) Na- 
tive and Alien Herbaceous Plants in the Brazilian Cerrado Are (Co-)Limited by Different Nutrients. Plant and Soil, 400, 231-243.

https://doi.org/10.1007/s11104-015-2725-9

[9] Lannes, L.S., Bustamante, M.M.C., Edwards, P.J. and Venterink, H.O. (2012) Alien and Endangered Plants in the Brazilian Cerrado Exhibit Contrasting Relationships with Vegetation Biomass and N :P Stoichiometry. New Phytologist, 196, 816-823. https://doi.org/10.1111/j.1469-8137.2012.04363.x

[10] Bustamante, M.M.C., de Brito, D.Q., Kozovits, A.R., Luedemann, G., de Mello, T.R.B., de Siqueira Pinto, A., Munhoz, C.B.R. and Takahashi, F.S.C. (2012) Effects of Nutrient Additions on Plant Biomass and Diversity of the Herbaceous-Subshrub Layer of a Brazilian Savanna (Cerrado). Plant Ecology, 213, 795-808. https://doi.org/10.1007/s11258-012-0042-4

[11] Novais, R.F., Smyth, T.J. and Nunes, F.N. (2007) Fósforo. In: Novais, R.F., Alvarez Venegas, V.H., Barros, N.F. de, Fontes, R.L.F., Cantarutti, R.B. and Neves, J.C.L., Eds., Fertilidade do Solo, 1st ed., Sociedade Brasileira de Ciência do Solo, Viçosa, MG, Brazil, 471-552.

[12] Taiz, L. and Zeiger, E. (2010) Plant Physiology. Taiz, L. and Zeiger, E., Eds., 5th ed., Sinauer Associates, Inc, Sunderland, MA, USA.

[13] Gonçalves, W.V., Vieira, M. do C., Heredia Zárate, N.A., Luciano, A.T., Rodrigues, W.B. and Tabaldi, L.A. (2010) Fósforo e Cama-de-Frango Semidecomposta na Produção de Carobinha (Jacaranda decurrens subsp. symmetrifoliolata). Horticultura Brasileira, 2, 3215-3220.

http://www.abhorticultura.com.br/eventosx/trabalhos/ev_4/A2536_T5120_Comp.p df

[14] Cantarella, H. (2007) Nitrogênio. In: Novais, R.F. de, Venegas, V.H.A., Barros, N.F. De, Fontes, R.L., Cantarutti, R.B. and Neves, J.C.L., Eds., Fertilidade do Solo, 1st ed., Sociedade Brasileira de Ciência do Solo, Viçosa, MG, Brazil, 375-470.

[15] Souza, S.R. and Fernandes, M.S. (2006) Nitrogênio. In: Fernandes, M.S., Ed., Nutrição Mineral de Plantas, 1st ed., Sociedade Brasileira de Ciência do Solo, Viçosa, MG, Brazil, 214-252.

[16] Chien, S.H., Gearhart, M.M. and Collamer, D.J. (2008) The Effect of Different Ammonical Nitrogen Sources on Soil Acidification. Soil Science, 173, 544-551. https://doi.org/10.1097/SS.0b013e31817d9d17

[17] Razaq, M., Zhang, P., Shen, H. and Salahuddin (2017) Influence of Nitrogen and Phosphorous on the Growth and Root Morphology of Acer mono. PLoS ONE, 12, e0171321. https://doi.org/10.1371/journal.pone.0171321

[18] Ahmed, S. and Hassan, K. (2015) Effect of Magnetic Water and Phosphorus Rates on Some Nutrients Uptake by Summer Squash Grown in Calcareous Soil of Duhok Governorate. Science Journal of University of Zakho, 3, 250-260. https://doi.org/10.25271/2015.3.2.46

[19] R Core Team. (2016) R: A Language and Environment for Statistical Computing. R Foundation for Statistical Computing, Vienna. https://www.r-project.org/

[20] Zeviani, W.M. and Serafim, M.E. (2016) EACS: Estatística Aplicada à Ciência do Solo. http://gitlab.c3sl.ufpr.br/walmes/EACS

[21] Venables, W.N. and Ripley, B.D. (2002) Modern Applied Statistics with S. Modern Applied Statistics with S, Springer New York, New York, NY. https://doi.org/10.1007/978-0-387-21706-2

[22] Pinheiro, J., Bates, D., DebRoy, S., Sarkar, D. and R Core Team. (2016) Nlme: Linear and Nonlinear Mixed Effects Models. http://cran.r-project.org/package=nlme 
[23] Sarkar, D. (2008) Lattice: Multivariate Data Visualization with R. Springer, New York, NY. https://doi.org/10.1007/978-0-387-75969-2

[24] Wickham, H. (2009) ggplot2. Springer, New York, NY. https://doi.org/10.1007/978-0-387-98141-3

[25] Hill, A. V. (1910) A New Mathematical Treatment of Changes of Ionic Concentration in Muscle and Nerve under the Action of Electric Currents, with a Theory as to Their Mode of Excitation. The Journal of Physiology, 40, 190-224. https://doi.org/10.1113/jphysiol.1910.sp001366

[26] Riley, D. and Barber, S. a. (1971) Effect of Ammonium and Nitrate Fertilization on Phosphorus Uptake as Related to Root-Induced $\mathrm{pH}$ Changes at the Root-Soil Interface1. Soil Science Society of America Journal, 35, 301. https://doi.org/10.2136/sssaj1971.03615995003500020035x

[27] Rachid-Edwards, M. (1956) Alguns Dispositivos para Proteção de Plantas Contra a Seca e o Fogo. Boletim da Faculdade de Filosofia, Ciências e Letras, Universidade de São Paulo. Botânica, 13, 35-69. https://doi.org/10.11606/issn.2318-5988.v13i0p35-69

[28] Alves, R.J. V, Da Silva, N.G., Fernandes Júnior, A.J. and Guimarães, A.R. (2013) Longevity of the Brazilian Underground Tree Jacaranda decurrens Cham. Anais da Academia Brasileira de Ciencias, 85, 671-677. https://doi.org/10.1590/S0001-37652013005000038

[29] Sangalli, A., Vieira, M.C., Heredia Zárate, N.A. and Scalon, S.P.Q. (2011) Desenvolvimento e Produção da Carobinha (Jacaranda decurrens Cham. Subsp. symmetrifoliolata Farias e Proença) Cultivada sob Dois Arranjos de Plantas, com ou sem Cobertura de Cama-de-Frango no Solo. Revista Brasileira de Plantas Medicinais, 13, 439-446. https://doi.org/10.1590/S1516-05722011000400009

[30] Barker, A. and Bryson, G. (2006) Nitrogen. In: Barker, A. V. and Pilbeam, D.J., Eds., Handbook of Plant Nutrition, CRC Press, 21-50. https://doi.org/10.1201/9781420014877.sec2

[31] Gouvea, A.B., Vieira, M.C., Heredia Z., N.A. and Pinto, J.V.C. (2014) Influência da Densidade de Plantio e da Cama de Frango na Produção da Carobinha (Jacaranda decurrens Cham. ssp. symmetrifoliolata Farias \& Proença). Revista Brasileira de Plantas Medicinais, 16, 481-489. https://doi.org/10.1590/1983-084X/09_167

[32] Moreira, D.G., Vieira, M.C., Heredia Zárate, N.A., Carnevali, T.O., Torales, E.P., Tabaldi, L.A., Lourente, E.R.P. and Mercante, F.M. (2016) Produtividade de Vinagreira, Pimenta Rosa e Carobinha Cultivadas em Sucessão a Mucuna Preta e Feijão de Porco. Revista Brasileira de Plantas Medicinais, 18, 326-335. https://doi.org/10.1590/1983-084X/15_085

[33] Jarrell, W.M. and Beverly, R.B. (1981) The Dillution Effect in Plant Nutrition Studies. Advances in Agronomy, 34, 197-224. https://doi.org/10.1016/S0065-2113(08)60887-1

[34] Lynch, J. (1995) Root Architecture and Plant Productivity. Plant Physiology, 109, 7-13. https://doi.org/10.1104/pp.109.1.7

[35] Han, M., Okamoto, M., Beatty, P.H., Rothstein, S.J. and Good, A.G. (2015) The Genetics of Nitrogen Use Efficiency in Crop Plants. Annual Review of Genetics, 49, 269-289. https://doi.org/10.1146/annurev-genet-112414-055037

[36] Bailey, S.J., Fulford, J., Vanhatalo, A., Winyard, P.G., Blackwell, J.R., DiMenna, F.J., Wilkerson, D.P., Benjamin, N. and Jones, A.M. (2010) Dietary Nitrate Supplementation Enhances Muscle Contractile Efficiency during Knee-Extensor Exercise in Humans. Journal of Applied Physiology, 109, 135-148. 
https://doi.org/10.1152/japplphysiol.00046.2010

[37] Hord, N.G. and Conley, M.N. (2017) Regulation of Dietary Nitrate and Nitrite: Balancing Essential Physiological Roles with Potential Health Risks. In: Bryan, N.S. and Loscalzo, J., Eds., Nitrite and Nitrate in Human Health and Disease, Springer International Publishing, Cham, 153-162.

https://doi.org/10.1007/978-3-319-46189-2_12 